\title{
FOURIER COEFFICIENTS OF EISENSTEIN SERIES OF THE EXCEPTIONAL GROUP OF TYPE $\mathrm{G}_{2}$
}

\author{
Dihua Jiang And Stephen Rallis
}

Let $F$ be a number fields and $K$ be a commutative algebra over $F$ of degree $n$. A basic question in number theory is whether the ratio $\frac{\zeta_{K}(s)}{\zeta_{F}(s)}$ of the two Dedekind zeta functions is an entire function in the complex variable $s$. From the point of view of the trace formula, the above basic question is expected to be equivalent to a basic question in automorphic L-functions, which asks whether or not the ratio $\frac{L^{S}\left(\Pi \otimes \Pi^{\vee}, s\right)}{\zeta_{F}^{S}(s)}$ is entire for all irreducible cuspidal automorphic representation of $G L\left(n, \mathbb{A}_{F}\right)$ with trivial central character, where $L^{S}\left(\Pi \otimes \Pi^{\vee}, s\right)$ is the standard tensor product $L$-function of $\Pi$ with its contragredient $\Pi^{\vee}$, see for example the work of Jacquet and Zagier [JaZa]. The main idea in this paper is to develop two intrinsically related methods to attack the above two questions. The work of Siegel [Sie], and of Shimura [Shi] (and of Gelbart and Jacquet [GeJa]) provided an evidence for this approach for the case of $n=2$. Combined with the work of Ginzburg [Gin], the main result of this paper shows that our approach works for the case of $n=3$. It is hoped that such an approach extends to at least the case of $n=5$.

\section{Introduction.}

We let $K$ and $F$ be number fields so that $K / F$ is a finite extension. We let $\zeta_{K}(s)$ and $\zeta_{F}(s)$ be the associated Dedekind zeta functions. A basic question is whether the ratio $\frac{\zeta_{K}(s)}{\zeta_{F}(s)}$ is an entire function in the complex variable $s$.

The first approach to analyzing such a question is connected to the use of Artin $L$-functions. For this we let $\Omega$ be a finite Galois extension of $F$ so that $\Omega \supseteq K \supseteq F$. If $G=\operatorname{Gal}(\Omega / F)$ and $H=\operatorname{Gal}(\Omega / K)$ are the associated Galois groups of $\Omega / F$ and $\Omega / K$, then we let $\sigma=\operatorname{ind}_{H}^{G}(1)$ be the induced module of $H$ to $G$ coming from the identity character on $H$. Then we consider the various irreducible $G$ modules $\sigma_{i}$ (with multiplicity $m_{i}$ ) that occur in $\sigma$, and we have that

$$
\zeta_{K}(s)=\prod L\left(s, \sigma_{i}\right)^{m_{i}}
$$

where $L\left(s, \sigma_{i}\right)$ is the associated Artin $L$-function determined by $\sigma_{i}$. We note that if $\sigma_{1}$ is the identity representation of $G$, then $m_{1}=1$, and the question 
whether $\frac{\zeta_{K}(s)}{\zeta_{F}(s)}$ is entire reduces to whether $\prod_{i>1} L\left(s, \sigma_{i}\right)^{m_{i}}$ is entire. We note that if $K / F$ is Galois, then by use of Brauer's basic theory $\frac{\zeta_{K}(s)}{\zeta_{F}(s)}$ is entire [Bra]. In fact, by use of the Artin conjecture (where $L\left(s, \sigma_{i}\right)$ is assumed to be entire for $i>1$ ) we would have that $\frac{\zeta_{K}(s)}{\zeta_{F}(s)}$ is also entire.

It may, however, be possible to prove that $\frac{\zeta_{K}(s)}{\zeta_{F}(s)}$ is entire without proving the Artin conjecture. Further discussion on the classical approach to this basic problem and its connection with the Artin conjecture can be found in J. Martinet's paper and R. van der Waall's paper in [Fro].

At the formal level, another approach to this question comes from simple considerations based on trace formula techniques. We consider the group $G L(n, F)$ and the associated adelized group $G L\left(n, \mathbb{A}_{F}\right)$. Let $\Phi$ be a $C^{\infty}$ function on $G L\left(n, \mathbb{A}_{F}\right)$ which is left and right invariant under the standardly chosen maximal compact subgroup of $G L\left(n, \mathbb{A}_{F}\right)$, transforms by the trivial character under the center $Z_{F}(\mathbb{A})$, and has a compact support modulo $Z_{F}(\mathbb{A})$. To such a function $\Phi$, we form the associated convolution kernel

$$
K_{\Phi}(x, y)=\sum_{\gamma \in G L(n, F) / Z_{F}(F)} \Phi\left(x^{-1} \gamma y\right)
$$

Then, formally, we compute the automorphic trace of $K_{\Phi}$ as follows:

$$
\int_{Z_{F}\left(\mathbb{A}_{F}\right) G L(n, F) \backslash G L\left(n, \mathbb{A}_{F}\right)} K_{\Phi}(x, x) d x .
$$

A basic method of computing such a family of integrals is through the use of truncation. Another method is to regularize the above family with an Eisenstein series $E_{0}(g, s)$ coming from the mirabolic subgroup (see [JaZa] for precise definitions, etc.). That is, we construct

$$
\int_{Z_{F}\left(\mathbb{A}_{F}\right) G L(n, F) \backslash G L\left(n, \mathbb{A}_{F}\right)} K_{\Phi}(x, x) E_{0}(x, s) d x .
$$

We note that when we formally unwind this integral (via the usual RankinSelberg method) we get two expansions, the spectral and the geometric. The spectral terms involve (at least for the cuspidal contribution) data of the form

$$
L^{S}\left(\Pi \otimes \Pi^{\vee}, s\right) a_{S}(s),
$$

where $\Pi$ is an irreducible cuspidal automorphic representation of $G L\left(n, \mathbb{A}_{F}\right)$ with trivial central character, $L^{S}\left(\Pi \otimes \Pi^{\vee}, s\right)$ is the standard tensor product L-function of $\Pi$ with its contragredient $\Pi^{\vee}, a_{S}(s)$ is a finite Euler product of Whittaker type integrals (see [JaSh]) and $S$ a finite set of primes (where 
$S$ is chosen appropriately). The geometric data involves (at least for those conjugacy classes on $G L(n, F)$ which are regular elliptic) terms of the form

$$
\zeta_{\widetilde{K}}^{S}(s) b_{S}(s),
$$

where $\widetilde{K}$ is some extension of $F$ of degree $n$ (not necessarily Galois) and $b_{S}(s)$ represents again a finite Euler product of Tate-like local integrals. The basic point here (at the formal level) is that there must be some relation between the data

$$
\left\{L^{S}\left(\Pi \otimes \Pi^{\vee}, s\right) \mid \Pi \text { cuspidal with trivial central character }\right\}
$$

and the data

$$
\left\{\zeta_{\widetilde{K}}^{S}(s) \mid \widetilde{K} \text { all commutative semisimple algebras of degree } n\right\} .
$$

The results of $[\mathbf{J a Z a}]$ suggest that there is a qualitative connection between the following two assertions:

(*) The $L$ function $L^{S}\left(\Pi \otimes \Pi^{\vee}, s\right)$ is divisible by $\zeta_{F}^{S}(s)$ for all cuspidal $\Pi$ (with trivial central character).

(**) The Dedekind zeta function $\zeta_{\widetilde{K}}^{S}(s)$ is divisible by $\zeta_{F}^{S}(s)$ for all $\widetilde{K}$, extensions of degree $n$ of $F$.

We note that in $[\mathrm{JaZa}]$ the precise form of the divisibility of the various terms appearing in the geometric expansion of $\left(^{* *}\right)$ by $\zeta_{\mathbb{Q}}(s)$ (in the $G L_{2}$ case) is proved. Also in [Fli], similar more general issues were considered.

We note that $L^{S}\left(\Pi \otimes \Pi^{\vee}, s\right)=\zeta_{F}^{S}(s) \cdot L^{S}(\Pi, \mathrm{Ad}, s)$ where $L^{S}(\cdot, \mathrm{Ad}, s)$ is the adjoint $L$ function. Thus $(*)$ reduces to the condition that $L^{S}(\Pi, \mathrm{Ad}, s)$ be entire for all cuspidal $\Pi$.

In the present work, we present another approach to the relation between $(*)$ and $(* *)$.

In the abstract, the method is the following. Suppose that $G$ is a reductive group with a maximal parabolic $P$ so that the adelically induced module $\operatorname{ind}_{P(\mathbb{A})}^{G(\mathbb{A})}\left(\delta_{P}^{\frac{1}{2}+s}\right)$ produces a family of Eisenstein series $E\left(f_{s}, \cdot\right)$ with the following basic properties. There exists parabolic subgroups $P_{1}$ and $P_{2}$ in the same standard position as $P$ with $V_{P_{1}}$ and $V_{P_{2}}$ the corresponding unipotent radicals of $P_{1}$ and $P_{2}$, satisfying the following conditions:

(i) A certain subset of unitary characters on $V_{P_{1}}\left(\mathbb{A}_{F}\right) / V_{P_{1}}(F)$ is indexed by the set of isomorphism classes of commutative semisimple algebras of degree $n$. (Recent work [WrYu] shows that (i) is possible in certain cases.)

(ii) There exists a unipotent subgroup $V_{2}$ of $V_{P_{2}}$ and a character $\psi_{\Lambda}$ on $V_{2}\left(\mathbb{A}_{F}\right) / V_{2}(F)$ so that $\operatorname{Stab}_{M_{P_{2}}}\left(\psi_{\Lambda}\right)$ is a subgroup of $M_{P_{2}}$ contains 
$S L(n, F)$. (Here $M_{P_{2}}$ is the Levi component of $P_{2}$ leaving $V_{2}$ stable by adjoint action.)

Then the desired properties of the family $E\left(f_{s}, \cdot\right)$ are given by:

(a) If $\widetilde{K}$ is a representative of an equivalence class of a $n$-th degree extension of $F$ (with $\psi_{\widetilde{K} / F}$ the associated character on $V_{1}\left(\mathbb{A}_{F}\right)$ ), then, up to a finite Euler factor, the Fourier coefficient

$$
\int_{V_{P_{1}}(F) \backslash V_{P_{1}}\left(\mathbb{A}_{F}\right)} E^{*}\left(f_{s}, \mu_{1}\right) \psi_{\widetilde{K} / F}\left(\mu_{1}\right) d u
$$

equals

$$
\frac{\zeta_{\widetilde{K}}^{S}(s)}{\zeta_{F}^{S}(s)}
$$

(b) If $f_{\Pi} \in \Pi$, a cuspidal representation of $G L\left(n, \mathbb{A}_{F}\right)$ with trivial central character, then, up to a finite Euler factor, the integral

$$
\int_{\left(V_{2}(F) \backslash V_{2}\left(\mathbb{A}_{F}\right)\right) \times\left(S L(n, F) \backslash S L\left(n, \mathbb{A}_{F}\right)\right)} E^{*}\left(f_{s}, \mu_{2} \cdot x\right) \psi_{\Lambda}\left(\mu_{2}\right) f_{\Pi}(x) d \mu_{2} d x
$$

equals

$$
L^{S}(\Pi, A d, s) .
$$

In cases (a) and (b), $E^{*}\left(f_{s}, \cdot\right)$ is the "normalized" Eisenstein series (so that $E^{*}\left(f_{s}, \cdot\right)$ has a finite number of poles in $\left.s\right)$.

Also, we note in (b) that we may have to replace $\psi_{\Lambda}$ by a $\theta$ function (Fourier Jacobi example).

These two conditions thus should possibly relate again $(*)$ and $(* *)$.

We note here that the existence of cases (a) and (b) above should allow us to prove directly the validity of $(*)$ and $(* *)$ separately. This would follow just from the analytic properties of the family of the normalized Eisenstein series $E^{*}\left(f_{s}, \cdot\right)$ and analytic properties of the local integrals in (a) and (b). We emphasize this point as the difference between this method and the second above. The latter case just asserts the equivalence of $(*)$ and $\left({ }^{*}\right)$.

The point of this paper is to show that this phenomenon works in the case where $n=3$. We note that the $n=2$ case basically is contained in the work of Siegel and Shimura. In that case, the group $G=G S p_{2}$, the rank 2 group of symplectic similitudes, and $P$ is the Siegel parabolic subgroup of $G$ with $P=P_{1}$ and $P_{2}$, the other maximal parabolic subgroup of $G S p_{2}$. We note the Fourier coefficients in (a) were first computed by Siegel in [Sie] and the corresponding Rankin-Selberg integral in (b) (which is a Fourier Jacobi case) was given classically by Shimura in [Shi] (generalized by Gelbart and Jacquet in [GeJa]). 
It is hoped that such a method extends to at least the case $n=5$.

In this paper we are providing evidence for this third approach to relate $(*)$ and $\left({ }^{*}\right)$. We expect this approach to yield some interesting arithmetic information.

We take in this paper the group $G$ to be the exceptional group $G_{2}$. We consider the maximal parabolic subgroup $P=M N$ associated to the short simple root $\beta$. Then $M \cong G L(2)$ (attached to $\beta$ ) and $N$ is a five dimensional unipotent subgroup of Heisenberg type with the center $Z$. Let $I(s):=\operatorname{ind}_{P(\mathbb{A})}^{G_{2}(\mathbb{A})}\left(\delta_{P}^{s}\right)$ be the unnormalized smooth induction from the modular character $\delta_{P}$ of $P$. To each section $f_{s} \in I(s)$, we define an Eisenstein series $E\left(g, s ; f_{s}\right)$ in the usual sense. We remark that it is this Eisenstein series used by D. Ginzburg in [Gin] to construct a Rankin-Selberg integral which represents the adjoint L-function of $G L(3)$. In other words, this Eisenstein series enjoys property (b) above. $E\left(g, s ; f_{s}\right)$ has the normalizing factor $\zeta(3 s) \zeta(6 s-2) \zeta(9 s-3)$. We denote the normalized Eisenstein series by $E^{*}\left(g, s ; f_{s}\right)$. We shall be concerned with the Fourier coefficients of the normalized Eisenstein series

$$
E^{*}\left(g, s ; f_{s}\right)=\zeta(3 s) \zeta(6 s-2) \zeta(9 s-3) E\left(g, s ; f_{s}\right)
$$

with respect to certain additive characters $\psi$ of $N(\mathbb{A}) / N(F)$ and prove that the Eisenstein series possesses property (a) above. The choice of these characters is based on the canonical geometrical structure on $N / Z$, which may be described as follows.

Under the adjoint action of $G L(2)$ on $V:=N / Z,(G L(2), V)$ carries a structure of a prehomogeneous vector space of parabolic type. The rational structure of $G L(2, F)$-orbits on $V(F)$ was explicitly given in [Wri] and $[\mathrm{DaWr}]$. The point here is that there exists a canonical one to one correspondence between the set of all $G L(2, F)$-orbits on $V(F)$ of maximal dimension (i.e. with the stabilizers being finite) and the set of isomorphism (over $F$ ) classes of all three dimensional commutative algebras $K$ over $F$. This statement holds when the base field $F$ is a global, local or finite field. To each $G L(2, F)$-orbit $\sigma$ on $V(F)$, we can canonically associate an additive unitary character $\psi_{\sigma}$ of $N(\mathbb{A}) / Z(\mathbb{A})$ (hence of $N(\mathbb{A})$ ).

Our main Theorem in this paper is that the Fourier coefficient of the normalized Eisenstein series $E^{*}\left(g, s ; f_{s}\right)$ with respect to the character $\psi_{\sigma}$ gives the ratio $\frac{\zeta_{K}(3 s-1)}{\zeta_{F}(3 s-1)}$, where $K$ is the three dimensional commutative algebra over $F$ corresponding to the $G L(2, F)$-orbit $\sigma$ on $V(F)$.

We note that the proof of $\left(^{*}\right)$ and $(* *)$ in this case requires a careful analysis of the global poles of the given Eisenstein series and of the local poles of certain zeta integrals as studied by Ginzburg in [Gin] and as the integrals given by formula (16) in Section 3 of this paper. 
We shall describe briefly the content of this paper. We first recall some known facts about the group $G_{2}$ from [Ste] and [GRS], and about the rational structure of the prehomogeneous space $(G L(2), V)$ from [Wri] and $[\mathrm{DaWr}]$. Then we prove $\S 3$ the Fourier coefficient under consideration is Eulerian. Correspondingly, the proof of local (p-adic) uniqueness property for the relevant (local) functionals will be given in $\S 4$. The most technical portion of the paper is $\S 5$, where we make the computation of the local integral with unramified data for three types of orbits $(0,1,1,0),(0,1,0, a)$, and $(1,0,0, a)$. In the last case, we assume that the local field $F_{v}$ contains the third root of unity. In this case, the computation of the local unramified integral is reduced to a precise evaluation of a multivariable cubic exponential sum (Lemma 2), which will be completed in the last section.

Based on the local unramified calculation, we can prove the main theorem (Theorem 2) in $\S 6$. The idea here is to make certain global-local relation work. Note that an orbit $\sigma=(1,0, c, d)$ over the global field $F$ may locally live in an orbit which has a representative of one of the three typical types as above. According to the canonical correspondence between the 'orbits' and the 'algebras' stated above, we can easily find an element $g_{v} \in G L\left(2, F_{v}\right)$ such that $g_{v} \circ(1,0, c, d)$ will be equal to one of $(0,1,1,0),(0,1,0, a)$, and $(1,0,0, a)$. The point is that for a given global orbit $(1,0, c, d)$, we can choose $g_{v} \in G L\left(2, \mathcal{O}_{v}\right)$ for all finite place $v$ of $F$ outsider a finite set $S$, which is determined by the global orbit $(1,0, c, d)$. It is evident that the Fourier coefficients of the local unramified section $f_{s, v}$ with respect to characters $\psi_{\sigma}$ are equal when $\sigma$ runs over a $G L\left(2, \mathcal{O}_{v}\right)$-orbit. It should be pointed out that in the fourth case of the main Theorem, we assume that the global base field $F$ contains the third root of unity. This assumption makes our computation easier. However, we believe that this assumption should not be essential to the statement of our main Theorem.

The project of this paper was initiated when both of the authors participated in the special year program of automorphic forms at the Mathematical Sciences Research Institute, Berkeley, 1994-95. We would like to thank the MSRI for hospitality. The first author is partly supported by the NSF Mathematical Research Fellowship. Finally we would like to thank the referee for kind comments and suggestions.

\section{Basic Structure of the Group $\mathrm{G}_{2}$.}

We shall recall some basic facts about the structure of the group $G_{2}$ from [Ste], [Car], and [GRS], on which our results will be built. Let $F$ be a field. We set $G_{2}=G_{2}(F)$. 
2.1. Root System of $\mathbf{G}_{2}$. Let $\Phi$ be the root system of the group $G_{2}$. Then one has $\Phi=\Phi^{+} \cup-\Phi^{+}$and

$$
\Phi^{+}:=\{\alpha, \beta, \alpha+\beta, \alpha+2 \beta, \alpha+3 \beta, 2 \alpha+3 \beta\}
$$

where $\alpha$ is the long simple root and $\beta$ is the short simple root, i.e., $\alpha$ and $\beta$ satisfy

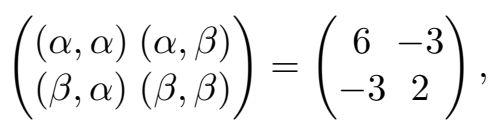

where $($,$) is the usual inner product in the vector space generated by these$ two simple roots. The Cartan matrix is

$$
\left(\begin{array}{c}
\langle\alpha \mid \alpha\rangle\langle\alpha \mid \beta\rangle \\
\langle\beta \mid \alpha\rangle\langle\beta \mid \beta\rangle
\end{array}\right)=\left(\begin{array}{cc}
2 & -3 \\
-1 & 2
\end{array}\right)
$$

where $\langle\gamma \mid \delta\rangle=\frac{2(\gamma, \delta)}{(\delta, \delta)}$. The Weyl group $W$ is the dihedral group of order 12, which is generated by the reflections $w_{\alpha}$ and $w_{\beta}$, with relations

$$
w_{\alpha}^{2}=w_{\beta}^{2}=\left(w_{\alpha} w_{\beta}\right)^{6}=1
$$

Precisely, one has

$$
\begin{aligned}
W=\left\{1, w_{\alpha}, w_{\beta}, w_{\alpha \beta}, w_{\beta \alpha},\right. & \\
& \left.w_{\alpha \beta \alpha}, w_{\beta \alpha \beta}, w_{\alpha \beta \alpha \beta}, w_{\beta \alpha \beta \alpha}, w_{\alpha \beta \alpha \beta \alpha}, w_{\beta \alpha \beta \alpha \beta}, w_{\alpha \beta \alpha \beta \alpha \beta}\right\}
\end{aligned}
$$

where $w_{\alpha \beta}:=w_{\alpha} w_{\beta}$, and so on.

2.2. Structure Constants. Let $\mathfrak{g}_{2}$ be the Lie algebra of $G_{2}$. Then one has a Cartan decomposition

$$
\mathfrak{g}_{2}=\mathfrak{h} \oplus \sum_{\gamma \in \Phi} \mathfrak{g}_{\gamma}
$$

For each root $\gamma \in \Phi$, fix a nonzero element $\mathbf{x}_{\gamma} \in \mathfrak{g}_{\gamma}$ and let $\mathbf{h}_{\gamma}:=\frac{2 \gamma}{(\gamma, \gamma)}$ be the associated coroot. Then these elements satisfy the following conditions:

$$
\left[\mathbf{x}_{\gamma}, \mathbf{x}_{\delta}\right]= \begin{cases}\mathbf{h}_{\gamma}, & \text { if } \delta=-\gamma, \\ 0, & \text { if } \gamma+\delta \notin \Phi, \\ N_{\gamma, \delta} \mathbf{x}_{\gamma+\delta}, & \text { if } \gamma+\delta \in \Phi\end{cases}
$$

Correspondingly, one has, to each root $\gamma$, a one-parameter unipotent subgroup $\chi_{\gamma}(x)$ and a one-parameter semisimple subgroup $h_{\gamma}(t)$ in $G_{2}$. Let 
$w_{\gamma}(1):=\chi_{\gamma}(1) \chi_{-\gamma}(-1) \chi_{\gamma}(1)$ be the preimage in $G_{2}$ of the Weyl group element $w_{\gamma}$. In the following, we will also denote $w_{\gamma}(1)$ by $w_{\gamma}$ if there is no chance of confusion.

Following p. 150 in [Ste], one can choose a Chevalley basis

$$
\left\{\mathbf{h}_{\alpha}, \mathbf{h}_{\beta} ; \mathbf{x}_{\gamma}, \gamma \in \Phi\right\}
$$

so that

(1) the structure constants associated to the positive roots are $N_{\alpha, \beta}=$ 1, $N_{\alpha+\beta, \beta}=2, N_{\beta, \alpha+2 \beta}=N_{\alpha+\beta, \alpha+2 \beta}=3$ and $N_{\alpha, \alpha+3 \beta}=1$; (Other structure constants are uniquely determined by these, see, for example, Chapter 4 in [Car].)

(2) $w_{\alpha \beta}$ satisfies that

$$
w_{\alpha \beta} \cdot \mathbf{x}_{\gamma}=-\mathbf{x}_{w_{\alpha \beta}(\gamma)}
$$

for any root $\gamma \in \Phi$;

(3) if $w_{\alpha} \cdot \mathbf{x}_{\gamma}:=a_{\alpha, \gamma} \mathbf{x}_{w_{\alpha}(\gamma)}$, then we have, following Proposition 6.4.3 in $[\mathrm{Car}]$, that $a_{\alpha, \alpha}=-1, a_{\alpha, \beta}=1, a_{\alpha, \alpha+\beta}=-1, a_{\alpha, \alpha+2 \beta}=1, a_{\alpha, \alpha+3 \beta}=$ 1 , and $a_{\alpha, 2 \alpha+3 \beta}=-1$

The following commutator relations will be used in our computation in later sections.

$$
\begin{aligned}
\left(\chi_{\alpha+3 \beta}(t), \chi_{-\alpha-2 \beta}(u)\right) & =\chi_{\beta}(t u) \chi_{-2 \alpha-3 \beta}\left(-t u^{3}\right) \chi_{-\alpha-\beta}\left(-t u^{2}\right) \chi_{-\alpha}\left(t^{2} u^{3}\right) \\
\left(\chi_{\alpha+3 \beta}(t), \chi_{-2 \alpha-3 \beta}(u)\right) & =\chi_{-\alpha}(t u) \\
\left(\chi_{\beta}(t), \chi_{-\alpha-\beta}(u)\right) & =\chi_{-\alpha}(3 t u) \\
\left(\chi_{\alpha+2 \beta}(t), \chi_{-\alpha-\beta}(u)\right) & =\chi_{\beta}(-2 t u) \chi_{-\alpha}\left(3 t u^{2}\right) \chi_{\alpha+3 \beta}\left(3 t^{2} u\right) \\
\left(\chi_{\alpha+2 \beta}(t), \chi_{-2 \alpha-3 \beta}(u)\right) & =\chi_{-\alpha}\left(t^{3} u^{2}\right) \chi_{\beta}\left(-t^{2} u\right) \chi_{-\alpha-\beta}(t u) \chi_{\alpha+3 \beta}\left(t^{3} u\right) \\
\left(\chi_{2 \alpha+3 \beta}(t), \chi_{-\alpha-\beta}(u)\right) & =\chi_{-\alpha}\left(t u^{3}\right) \chi_{\alpha+3 \beta}\left(t^{2} u^{3}\right) \chi_{\alpha+2 \beta}(-t u) \chi_{\beta}\left(-t u^{2}\right) .
\end{aligned}
$$

These identities can be verified in terms of the structure constants chosen as above.

2.3. Subgroups. Let $B$ be the standard Borel subgroup of $G_{2}$ corresponding to the set of positive roots $\Phi^{+}$. Then $B=T U$. The maximal torus $T$ is of two dimension, the elements of which are denoted by $h\left(t_{1}, t_{2}\right)$ for $t_{1}, t_{2} \in F^{\times}$. Further, one has, according to the Cartan matrix,

$$
(a \alpha+b \beta) h\left(t_{1}, t_{2}\right)=\left(\frac{t_{1}}{t_{2}}\right)^{a}\left(t_{2}\right)^{b}
$$

for $a \alpha+b \beta \in \Phi$. To each root $\gamma \in \Phi$, we have that $\left\{h_{\gamma}(t), \chi_{\gamma}(u), \chi_{-\gamma}(u)\right\}$ generates a subgroup in $G_{2}$, which is isomorphic to $S L(2)$. Further we have 
$w h_{\gamma}(t) w^{-1}=h_{w(\gamma)}(t)$ for $w \in W$ and

$$
\begin{aligned}
& h_{\alpha}(t)=h\left(t, t^{-1}\right), h_{\beta}(t)=h\left(t^{-1}, t^{2}\right), h_{\alpha+\beta}(t)=h\left(t^{2}, t^{-1}\right), \\
& h_{\alpha+2 \beta}(t)=h(t, t), h_{\alpha+3 \beta}(t)=h(1, t), h_{2 \alpha+3 \beta}(t)=h(t, 1) .
\end{aligned}
$$

Let $P=M N$ be the standard maximal parabolic subgroup associated to the short simple root $\beta$. Then $M=G L(2)$ and $N$ is a five dimensional unipotent subgroup of Heisenberg type generated by the following positive roots

$$
\{\alpha, \alpha+\beta, \alpha+2 \beta, \alpha+3 \beta, 2 \alpha+3 \beta\} \text {. }
$$

The subgroup $Z:=\left\{\chi_{2 \alpha+3 \beta}(x)\right\}$ is the center. We choose the coordinates on $N$ as follows: for any $n \in N$, we set

$$
n:=n(x, y, z, u, v):=\chi_{\alpha+3 \beta}(x) \chi_{\alpha+2 \beta}(y) \chi_{2 \alpha+3 \beta}(z) \chi_{\alpha+\beta}(u) \chi_{\alpha}(v) .
$$

It is easy to check the following Bruhat decomposition of $G_{2}$ with respect to the parabolic subgroup $P$,

$$
G_{2}=[P] \cup\left[P w_{\alpha} P\right] \cup\left[P w_{\alpha \beta \alpha} P\right] \cup\left[P w_{\alpha \beta \alpha \beta \alpha} P\right]
$$

and also, if we set

(10) $n^{-}(x, y, z, u, v):=\chi_{-\alpha}(x) \chi_{-\alpha-\beta}(y) \chi_{-2 \alpha-3 \beta}(z) \chi_{-\alpha-2 \beta}(u) \chi_{-\alpha-3 \beta}(v)$,

then we have $n^{-}(x, y,-z,-u,-v)=w_{\alpha \beta \alpha \beta \alpha} \cdot n(x, y, z, u, v)$.

2.4. Certain Characters on the Unipotent Radical N. To define certain unitary characters of the unipotent radical $N$, we consider the adjoint action of the Levi subgroup $M$ on the unipotent radical $N$. Since the action keep the center $Z$ stable, we obtain an action of $G L(2)$ on the four dimensional vector space $V:=N / Z$. We notice that the pair $(G L(2), V)$ has a structure of irreducible regular prehomogeneous vector space in the sense of M. Saito.

For any character $\psi$ of $N / Z$, there exists a $\sigma \in V$, so that

$$
\psi \sigma(n)=\psi_{\circ}(\langle\sigma, n\rangle)
$$

for any $n \in N$, where the pairing $\langle\cdot, \cdot\rangle$ has property that

$$
\langle g \cdot \sigma, g \cdot n\rangle=\langle\sigma, n\rangle
$$

for any $g \in G L(2)$ and $\sigma, n \in V$. We will denote

$$
\psi_{\sigma}(\cdot)=\psi_{\circ}(\langle\sigma, \cdot\rangle)
$$


and extend $\psi_{\sigma}(\cdot)$ to be a character of the unipotent radical $N$. When the field $F$ is a number field or its local completion, the parameterization of the $F$-rational $G L(2, F)$-orbits on $V(F)$ is given in [Wri] and [DaWr]. The representatives of $F$-rational $G L(2, F)$ nonsingular orbits on $V(F)$ are of following three types: $\sigma_{1}=(0,1,1,0), \sigma_{2}=(0,1,0, a)$, and $\sigma_{3}=(1,0, b, c)$, where $a, b, c \in F$ satisfy the condition that the polynomials $x^{2}+a$ and $x^{3}+b x-c$ are irreducible over $F$. For these typical representatives of the nonsingular orbits, we may make the associated characters more precise:

$$
\begin{aligned}
& \psi_{\sigma_{1}}(n(x, y, z, u, v))=\psi_{\circ}(u+y) \\
& \psi_{\sigma_{2}}(n(x, y, z, u, v))=\psi_{\circ}(a v+y) \\
& \psi_{\sigma_{3}}(n(x, y, z, u, v))=\psi_{\circ}(c v+b u+x)
\end{aligned}
$$

\section{Fourier Coefficients of Eisenstein Series.}

Let $F$ be a number field and $\mathbb{A}=\mathbb{A}_{F}$ the adele ring of $F$. For $p \in P$, let $\delta_{P}(p):=|\operatorname{det} \operatorname{Ad}(p)|_{\mathfrak{n}} \mid$, where $\mathfrak{n}$ is the Lie algebra of the unipotent radical $N$. Then one has, for $h\left(t_{1}, t_{2}\right) \in T$,

$$
\delta_{P}\left(h\left(t_{1}, t_{2}\right)\right)=\left|t_{1}^{2} t_{2}\right|^{3} .
$$

Consider the (unnormalized) smooth induced representation $I_{P}(s):=$ $\operatorname{ind}_{P(\mathbb{A})}^{G_{2}(\mathbb{A})}\left(\delta_{P}^{s}\right)$. As usual, to each section $f_{s} \in I_{P}(s)$, one may associate an Eisenstein series

$$
E\left(g, s ; f_{s}\right):=\sum_{\gamma \in P(F) \backslash G_{2}(F)} f_{s}(\gamma g) .
$$

From the general theory of Eisenstein series, as a function in the complex variable $s, E\left(g, s ; f_{s}\right)$ converges absolutely for real part of $s$ large and has a meromorphic continuation to the whole $s$-plane. After certain normalization, the possible poles of the normalized Eisenstein series associated to $E\left(g, s ; f_{s}\right)$ are determined in [ $\mathbf{J i a}]$.

For a unitary character $\psi_{\sigma}$, the Fourier coefficient of an Eisenstein series is defined as follows,

$$
E_{\psi_{\sigma}}\left(g, s ; f_{s}\right):=\int_{N(F) \backslash N(\mathbb{A})} E\left(n g, s ; f_{s}\right) \psi_{\sigma}(n) d n
$$

where $d n$ is the normalized Haar measure on $N(F) \backslash N(\mathbb{A})$ as usual.

Theorem 1. Let $f_{s} \in I_{P}(s)$ be a factorizable section. Then the Fourier coefficient of the Eisenstein series $E\left(g, s ; f_{s}\right)$ with respect to a unitary character $\psi_{\sigma_{i}}$ of $N$ for $i=1,2,3,4$ are Eulerian. 
Proof. After unfolding the Eisenstein series, the Fourier coefficient becomes a sum of four terms, according to the Bruhat decomposition, that is,

$$
\begin{aligned}
E_{\psi_{\sigma}}\left(g, s ; f_{s}\right)= & \int_{N(F) \backslash N(\mathbb{A})} E\left(n g, s ; f_{s}\right) \psi_{\sigma}(n) d n \\
= & \sum_{\gamma \in P \backslash P} \int_{N(F) \backslash N(\mathbb{A})} f_{s}(\gamma n g) \psi_{\sigma}(n) d n \\
& +\sum_{\gamma \in P \backslash P w_{\alpha} P} \int_{N(F) \backslash N(\mathbb{A})} f_{s}(\gamma n g) \psi_{\sigma}(n) d n \\
& +\sum_{\gamma \in P \backslash P w_{\alpha \beta \alpha} P} \int_{N(F) \backslash N(\mathbb{A})} f_{s}(\gamma n g) \psi_{\sigma}(n) d n \\
& +\sum_{\gamma \in P \backslash P w_{\alpha \beta \alpha \beta \alpha} P} \int_{N(F) \backslash N(\mathbb{A})} f_{s}(\gamma n g) \psi_{\sigma}(n) d n .
\end{aligned}
$$

For and $w \in W$, one writes $N=N^{w} N_{w}$ as usual. It is clear that the first term is zero. The second and the third terms will be zero if $\psi_{\sigma}\left(\gamma^{-1} \cdot n\right) \not \equiv 1$ for all the $\gamma^{\prime}$ 's and for $n \in N^{w_{\alpha}}$ and $n \in N^{w_{\alpha \beta \alpha}}$, respectively.

First it is not difficult to check the following

$$
\begin{aligned}
P \backslash P w_{\alpha} P & =w_{\alpha}\left[B \backslash G L_{\beta}(2)\right] N_{w_{\alpha}} \\
P \backslash P w_{\alpha \beta \alpha} P & =w_{\alpha \beta \alpha}\left[B \backslash G L_{\beta}(2)\right] N_{w_{\alpha \beta \alpha}}
\end{aligned}
$$

as algebraic varieties. We thus obtain that the second term can be written as

$$
\begin{aligned}
& \sum_{\gamma \in B \backslash G L_{\beta}(2)} \int_{N_{w_{\alpha}}(\mathbb{A})} f_{s}\left(w_{\alpha} n_{1} \gamma g\right) \psi_{\sigma}\left(\gamma^{-1} \cdot n_{1}\right) d n_{1} \\
& \cdot \int_{N w_{\alpha}(F) \backslash N w_{\alpha}(\mathbb{A})} \psi_{\sigma}\left(\gamma^{-1} \cdot n_{2}\right) d n_{2},
\end{aligned}
$$

and the third term can be similarly expressed as

$$
\begin{aligned}
& \sum_{\gamma \in B \backslash G L_{\beta}(2)} \int_{N_{w_{\alpha \beta \alpha}(\mathbb{A})}} f_{s}\left(w_{\alpha \beta \alpha} n_{1} \gamma g\right) \psi_{\sigma}\left(\gamma^{-1} \cdot n_{1}\right) d n_{1}
\end{aligned}
$$

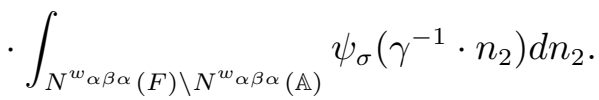

By Bruhat decomposition for $G L(2)$, it suffices to check the condition

$$
\psi_{\sigma}\left(\gamma^{-1} \cdot n\right) \not \equiv 1
$$


for $\gamma=w_{\beta}$ and $\gamma=\chi_{-\beta}(t)$. Let $n(x, y, z, u, v)$ be an element in $N$ as in (8), $\S 2.3$. Then one can easily check that

$$
w_{\beta} \cdot n(x, y, z, u, v)=n(-v,-u, p(z, x, y, u, v), y, x)
$$

and

$$
\begin{aligned}
\chi_{\beta}(t) \cdot n(x, y, z, u, v)= & n\left(x, y+a_{1} x t, z+a_{4} x^{2} t^{3}+b_{2} y^{2} t,\right. \\
& \left.u+b_{1} y t+a_{2} x t^{2}, v+c u t+b_{3} y t^{3}+a_{3} x t^{3}\right)
\end{aligned}
$$

where $a_{i}= \pm 1, b_{j}= \pm 2$ or \pm 3 , and $c= \pm 3$. Because

$$
\begin{aligned}
N^{w_{\alpha}} & =\{n(x, y, z, u, 0): x, y, z, u \in F\} \\
N^{w_{\alpha \beta \alpha}} & =\{n(x, y, 0,0,0): x, y \in F\},
\end{aligned}
$$

the nontriviality condition $\psi_{\sigma}\left(\gamma^{-1} \cdot n\right) \not \equiv 1$ for $\gamma=w_{\beta}$ and $\gamma=\chi_{-\beta}(t)$ can be seen by straightforward computation. Therefore, we obtain that

$$
\begin{aligned}
E_{\psi_{\sigma_{i}}}\left(g, s ; f_{s}\right) & =\sum_{\gamma \in P \backslash P w_{\alpha \beta \alpha \beta \alpha} P} \int_{N(F) \backslash N(\mathbb{A})} f_{s}(\gamma n g) \psi_{\sigma_{i}}(n) d n \\
& =\int_{N(\mathbb{A})} f_{s}\left(w_{\alpha \beta \alpha \beta \alpha} n g\right) \psi_{\sigma_{i}}(n) d n \\
& =\prod_{v} \int_{N\left(F_{v}\right)} f_{s, v}\left(w_{\alpha \beta \alpha \beta \alpha} n g\right) \psi_{\sigma_{i}, v}(n) d n .
\end{aligned}
$$

We denote the local integral by

$$
I_{v}^{\sigma_{i}}\left(s, f_{s}\right):=\int_{N\left(F_{v}\right)} f_{s, v}\left(w_{\alpha \beta \alpha \beta \alpha} n\right) \psi_{\sigma_{i}, v}(n) d n .
$$

The main Theorem is

Theorem 2. Let $F$ be a number field. The Fourier coefficients of the Eisenstein series $E\left(g, s ; f_{s}\right)$ with respect to the additive unitary characters $\psi_{\sigma_{i}}$ for $i=1,2,3,4$ can be written in the following forms, respectively.

(1) When $\sigma_{1}=(0,1,1,0)$,

$$
E_{\psi_{\sigma_{1}}}\left(e, s ; f_{s}\right)=\frac{\left[\zeta_{F}^{S}(3 s-1)\right]^{2}}{\zeta_{F}^{S}(3 s) \zeta_{F}^{S}(6 s-2) \zeta_{F}^{S}(9 s-3)} \prod_{v \in S} I_{v}^{\sigma_{1}}\left(s, f_{s, v}\right),
$$

where $S$ is such a finite subset of places of $F$ that if $v \in S, v$ is finite and $f_{s, v}$ and $\psi_{v}$ are unramified. 
(2) When $\sigma_{2}=(0,1,0, a)$,

$$
E_{\psi_{\sigma_{2}}}\left(e, s ; f_{s}\right)=\frac{\zeta_{F(\sqrt{a})}^{S}(3 s-1)}{\zeta_{F}^{S}(3 s) \zeta_{F}^{S}(6 s-2) \zeta_{F}^{S}(9 s-3)} \prod_{v \in S} I_{v}^{\sigma_{2}}\left(s, f_{s, v}\right),
$$

where $S$ is such a finite subset of places of $F$ that if $v \in S, v$ is finite and $f_{s, v}$ and $\psi_{v}$ are unramified and $a$ is a unit at $v$.

(3) When $\sigma_{3}=(1,0,0, a)$,

$$
E_{\psi_{\sigma_{3}}}\left(e, s ; f_{s}\right)=\frac{\zeta_{F\left(a^{\frac{1}{3}}\right)}^{S}(3 s-1)}{\zeta_{F}^{S}(3 s) \zeta_{F}^{S}(3 s-1) \zeta_{F}^{S}(6 s-2) \zeta_{F}^{S}(9 s-3)} \prod_{v \in S} I_{v}^{\sigma_{3}}\left(s, f_{s, v}\right),
$$

where $S$ is such a finite subset of places of $F$ that if $v \in S, v$ is finite and $f_{s, v}$ and $\psi_{v}$ are unramified and $a$ is a unit at $v$.

(4) When $\sigma_{4}=(1,0, b, a)$, we assume that $F$ contains the third root of unity, and then we have

$$
E_{\psi_{\sigma_{4}}}\left(e, s ; f_{s}\right)=\frac{\zeta_{K}^{S}(3 s-1)}{\zeta_{F}^{S}(3 s) \zeta_{F}^{S}(3 s-1) \zeta_{F}^{S}(6 s-2) \zeta_{F}^{S}(9 s-3)} \prod_{v \in S} I_{v}^{\sigma_{4}}\left(s, f_{s, v}\right)
$$

where $K$ is a cubic extension of $F$ generated by one of the roots of the irreducible polynomial $x^{3}+b x-a$, and $S$ is a finite subset of places of $F$ determined by $a, b, f_{s}$, and $\psi$.

Remark 1. As mentioned in $\S 1, \zeta_{F}(3 s) \zeta_{F}(6 s-2) \zeta_{F}(9 s-3)$ is the normalizing factor for this family of Eisenstein series [Jia]. The theorem actually means that the Fourier coefficient of the normalized Eisenstein series with respect to the character $\psi_{\sigma}$ gives the ratio $\frac{\zeta_{K}(3 s-1)}{\zeta_{F}(3 s-1)}$, where $K$ is the three dimensional semisimple commutative algebra corresponding to the orbit $\sigma$.

In the fourth part of Theorem 2, we have an assumption that the base field $F$ contains the third root of unity. We believe that this assumption is made for some technical reasons and should not be essential.

Theorem 2 will be proved in $\S 6$ based on the results in $\S 5$.

\section{Local Uniqueness.}

Let $F$ be a nonarchimedean local field of characteristic zero. We shall prove the following local uniqueness property for the functional related to our local integral.

Theorem 3 (Local Uniqueness). Let $\sigma$ be a representative of a nonsingular orbit of $G L(2)$ on $N / Z$. Then the dimension of the space

$$
\operatorname{Hom}_{G_{2}}\left(\operatorname{ind}_{P(F)}^{G_{2}(F)}\left(\delta_{P}^{s}\right), \operatorname{ind}_{N(F)}^{G_{2}(F)}\left(\psi_{\sigma}\right)\right)
$$

is at most one. 
Proof. It is enough to prove the result for a specifically chosen representative of each nonsingular orbit, for instance, $\sigma_{i}$ for $i=1,2,3$ as in (12). By Frobenius Reciprocity Law and the standard argument, it suffices to prove that for $i=1,2,3$, the uniqueness of the distribution $\mathcal{T}_{i}$ on $G_{2}(F)$ satisfying the following quasi-invariant property:

$$
(p, n) \circ \mathcal{T}_{i}(\varphi)=\delta_{P}^{s}(p) \psi_{\sigma_{i}}^{-1}(n) \mathcal{T}_{i}(\varphi)
$$

for $\varphi \in \mathcal{S}\left(G_{2}(F)\right)$ and $p \in P, n \in N$. Equivalently, it is enough to prove the dimension of the the space of the general functions $T_{i}$ defined by

$$
\mathcal{T}_{i}(\varphi):=\int_{G_{2}(F)} \varphi(g) T_{i}(g) d g
$$

and with the following quasi-invariant property

$$
T_{i}(p g n)=\delta_{P}^{-s}(p) \psi_{\sigma_{i}}^{-1}(n) T_{i}(g)
$$

is at most one.

We shall first prove that there exist no such distributions on $G_{2}(F)$ with support in the union of all lower dimensional cells, i.e. in $G_{2}(F) \backslash P w_{\alpha \beta \alpha \beta \alpha} P$. This will imply the uniqueness we need since on the open cell $P w_{\alpha \beta \alpha \beta \alpha} P$ there is one such distribution up to a scalar.

It is sure that there are no such distributions having support in $P$ by the nontriviality of the additive character. The same reason works for distributions on $P w_{\alpha} P$ or on $P w_{\alpha \beta \alpha} P$. More precisely, we have

$$
\begin{aligned}
P w_{\alpha} P & =\left[P w_{\alpha} N\right] \cup\left[P w_{\alpha \beta} \chi_{\beta}(t) N\right] \\
P w_{\alpha \beta \alpha} P & =\left[P w_{\alpha \beta \alpha} N\right] \cup\left[P w_{\alpha \beta \alpha \beta} \chi_{\beta}(t) N\right] .
\end{aligned}
$$

By checking the roots in $N^{w_{\alpha}}$ and in $N^{w_{\alpha \beta \alpha}}$, we can easily figure out that any distribution with property (17) or (18) on $P w_{\alpha} N$ or on $P w_{\alpha \beta \alpha} N$ vanishes.

Similarly, we have

$$
\begin{aligned}
\left(\chi_{\beta}(t) N\right)^{w_{\alpha \beta}} & =\left\langle\chi_{\alpha}, \chi_{\alpha+\beta}, \chi_{\alpha+2 \beta}, \chi_{2 \alpha+3 \beta}\right\rangle \\
\left(\chi_{\beta}(t) N\right)^{w_{\alpha \beta \beta} \beta} & =\left\langle\chi_{\alpha}, \chi_{\alpha+\beta}\right\rangle .
\end{aligned}
$$

The distributions $T_{i}$ on $P w_{\alpha \beta} \chi_{\beta}(t) N$ or on $P w_{\alpha \beta \alpha \beta} \chi_{\beta}(t) N$ give rise to distributions $T_{i}$ on $\chi_{\beta}(t) \chi_{\alpha+\beta}(u)$ with following property

$$
\begin{aligned}
T\left(\chi_{\beta}(t) \chi_{\alpha+\beta}(u)\right) & =T\left(\chi_{\beta}(t)\right) \psi_{\sigma_{i}}^{-1}\left(\chi_{\alpha+\beta}(u)\right) \\
& =T\left(\chi_{\beta}(t)\right) \psi_{\sigma_{i}}^{-1}\left(\chi_{\alpha+3 \beta}\left(3 u t^{2}\right) \chi_{\alpha+2 \beta}(-2 u t)\right),
\end{aligned}
$$


because

$$
\chi_{\beta}(t) \chi_{\alpha+\beta}(u)=\chi_{\alpha+\beta}(u) \chi_{\beta}(t) \chi_{\alpha+3 \beta}\left(3 u t^{2}\right) \chi_{\alpha+2 \beta}(-2 u t) \chi_{2 \alpha+3 \beta}\left(3 u^{2} t\right) .
$$

The existence of such nonzero distributions on $\chi_{\beta}(t) \chi_{\alpha+\beta}(u)$, hence on $P w_{\alpha \beta} \chi_{\beta}(t) N$ or on $P w_{\alpha \beta \alpha \beta} \chi_{\beta}(t) N$ implies

$$
\psi_{\sigma_{i}}\left(\chi_{\alpha+\beta}(u)\right)=\psi_{\sigma_{i}}\left(\chi_{\alpha+3 \beta}\left(3 u t^{2}\right) \chi_{\alpha+2 \beta}(-2 u t)\right)
$$

for all $t, u \in F$. This is an obvious contradiction.

The nonexistence of such distributions on each piece implies the nonexistence of such distributions on their union since there are only finitely many pieces. This proves the theorem.

\section{Unramified Computation.}

In this section, we assume that $F$ is a nonarchimedean local field of characteristic zero. We shall compute the unramified local integral for each orbit separately.

5.1. Case: $\sigma_{3}=(1,0,0, a)$. In this subsection, we assume that the base field $F$ contains the third root of unity. We are going to compute the following integral

$$
I\left(f_{s}, \psi_{\sigma_{3}}\right)=\int_{N(F)} f_{s}\left(w_{\alpha \beta \alpha \beta \alpha} n(x, y, z, u, v)\right) \psi_{\circ}(a v+x) d n
$$

with unramified data $\left(f_{s}, \psi_{\circ}\right)$, that is, $f_{s}$ is the unramified section in $I_{P(F)}(s)$ so that $f_{s}(1)=1$ and $\psi_{\circ}$ is the unramified additive character of the field $F$. Recall from Section 2.3 that $w_{\alpha \beta \alpha \beta \alpha} \cdot n(x, y, z, u, v)=n^{-}(x, y,-z,-u,-v)$, where

$$
n^{-}(x, y, z, u, v):=\chi_{-\alpha}(x) \chi_{-\alpha-\beta}(y) \chi_{-2 \alpha-3 \beta}(z) \chi_{-\alpha-2 \beta}(u) \chi_{-\alpha-3 \beta}(v) .
$$

In other words, we shall compute the following integral

$$
I\left(f_{s}, \psi_{\sigma_{3}}\right)=\int_{F^{5}} f_{s}\left(n^{-}(x, y,-z,-u,-v)\right) \psi_{\circ}(a v+x) d n .
$$

We need some lemmas for our computation of the integral.

Lemma 1. Let $a \in F, s$ a complex number, and $e$ an integer. Then

$$
\begin{aligned}
& \int_{|x| \geq q^{e}}|x|^{-s} \psi_{\circ}(a x) d x \\
& = \begin{cases}0, & \text { if } e>\operatorname{ord}(a)+1, \\
\frac{q^{-e(s-1)}-q^{-(s-1)}|a|^{s-1}}{1-q^{-(s-1)}}\left(1-q^{-1}\right)-q^{-s}|a|^{s-1}, & \text { if } e \leq \operatorname{ord}(a)+1 .\end{cases}
\end{aligned}
$$


Lemma 2. Let $\psi_{\circ}$ be an unramified additive character and $a \in \mathcal{O}^{\times}$. Assume that $q \equiv 1(\bmod 3)$. Then

$\sum_{\xi, \zeta, \nu \in \mathbb{F}_{q}^{\times} ; \mu \in \mathbb{F}_{q}} \psi_{\circ}\left(\left[a \xi^{3}-\mu^{3}-\zeta^{2}+\zeta-3 \mu \zeta\right] \pi^{-1} \nu\right)= \begin{cases}0, & \text { if } a \not \equiv \alpha^{3}(\bmod \pi \mathcal{O}) \\ 3 q, & \text { if } a \equiv \alpha^{3}(\bmod \pi \mathcal{O}) .\end{cases}$

Remark 2. Lemma 1 follows from easy calculation. The proof of Lemma 2 will need more effort. The case when $a \equiv \alpha^{3}(\bmod \pi \mathcal{O})$ for some $\alpha \in \mathcal{O}^{\times}$will be proved as a corollary (Corollary 3 , in $\S 5.2$ ) of Proposition 6 and Corollary 2 in $\S 5$. The case when $a \not \equiv \alpha^{3}(\bmod \pi \mathcal{O})$ will be more complicated and proved in the last section.

We shall reduce the computation of integral $I\left(f_{s}, \psi_{\sigma_{3}}\right)$ by using Bruhat decomposition once for each variable. We introduce some notations for our reduction.

$$
\begin{aligned}
& I_{+}:=\int_{F^{4},|v| \leq 1} f_{s}\left(n^{-}(x, y,-z,-u,-v)\right) \psi_{\circ}(a v+x) d n \\
& I_{-}:=\int_{F^{4},|v|>1} f_{s}\left(n^{-}(x, y,-z,-u,-v)\right) \psi_{\circ}(a v+x) d n .
\end{aligned}
$$

It is easy to see that

$$
I_{+}=\int_{F^{4}} f_{s}\left(n^{-}(x, y,-z,-u)\right) \psi_{\circ}(x) d n
$$

since $f_{s}$ is $K$-invariant (where $K=G_{2}(\mathcal{O})$ ) and $a$ is a unit. When $|v|>1$, we have the following Bruhat decomposition related to the root $-\alpha-3 \beta$ :

$$
\chi_{-\alpha-3 \beta}(-v)=h_{\alpha+3 \beta}\left(v^{-1}\right) \chi_{\alpha+3 \beta}(-v) k_{\alpha+3 \beta}(v) .
$$

It follows from the facts stated in $\S 2$ that $h_{\alpha+3 \beta}\left(v^{-1}\right)=h\left(1, v^{-1}\right)$ and $\delta_{P}\left(h\left(1, v^{-1}\right)\right)=|v|^{-3}$, and also

$$
\begin{aligned}
& n^{-}(x, y,-z,-u) \chi_{-\alpha-3 \beta}(-v) \\
& =h\left(1, v^{-1}\right) n^{-}\left(x v, y,-z v^{-1},-u v^{-1}\right) \chi_{\alpha+3 \beta}(-v) k_{\alpha+3 \beta}(v) .
\end{aligned}
$$

Hence we obtain that

$$
I_{-}=\int_{F^{4},|v|>1} f_{s}\left(n^{-}(x, y,-z,-u) \chi_{\alpha+3 \beta}(-v)\right)|v|^{-3 s+1} \psi_{\circ}\left(a v+x v^{-1}\right) d n .
$$


Applying the commutator relations in $\S 2.2$, we have the following

$$
\begin{aligned}
& f_{s}\left(n^{-}(x, y,-z,-u) \chi_{\alpha+3 \beta}(-v)\right) \\
& =f_{s}\left(n^{-}\left(x-v z+3 v u y-u^{3} v^{2}, y-v u^{2},-z+v u^{3},-u\right)\right)
\end{aligned}
$$

and then

$$
I_{-}=\int_{F^{4},|v|>1} f_{s}\left(n^{-}(x, y,-z,-u)\right)|v|^{-3 s+1} \psi_{\circ}\left(a v-v u^{3}+x v^{-1}+z-3 u y\right) d n .
$$

Then we cut the domain of integration further and consider the case of $|u|>1$. We have $\chi_{-\alpha-2 \beta}(-u)=h_{\alpha+2 \beta}\left(u^{-1}\right) \chi_{\alpha+2 \beta}(-u) k_{\alpha+2 \beta}(u)$. Since $h_{\alpha+2 \beta}\left(u^{-1}\right)=h\left(u^{-1}, u^{-1}\right)$ and $h_{\alpha+2 \beta}\left(u^{-1}\right) \cdot n^{-}(x, y,-z)=n^{-}\left(x, u^{-1} y\right.$, $\left.-u^{-3} z\right)$, we have

$$
\begin{aligned}
I_{--}= & \int_{F^{3} ;|u|,|v|>1} f_{s}\left(n^{-}(x, y,-z) \chi_{\alpha+2 \beta}(-u)\right)|v|^{-3 s+1}|u|^{-9 s+4} \\
& \cdot \psi_{\circ}\left(a v-v u^{3}+x v^{-1}+z u^{3}-3 u^{2} y\right) d n .
\end{aligned}
$$

By the commutator relations in $\S 2.2$,

$$
f_{s}\left(n^{-}(x, y,-z) \chi_{\alpha+2 \beta}(-u)\right)=f_{s}\left(n^{-}\left(x+u^{3} z^{2}+3 u y(y-u z), y-u z,-z\right)\right) .
$$

Hence we obtain that

$$
\begin{aligned}
I_{--} & =\int_{F^{3} ;|u|,|v|>1} f_{s}\left(n^{-}(x, y,-z)\right)|v|^{-3 s+1}|u|^{-9 s+4} \\
& \cdot \psi_{\circ}\left(a v-v u^{3}+\left(x-u^{3} z^{2}-3 u y(y+u z)\right) v^{-1}-2 z u^{3}-3 u^{2} y\right) d n .
\end{aligned}
$$

For the variable $z$, when $|z|>1$, we similarly have the Bruhat decomposition $\chi_{-2 \alpha-3 \beta}(-z)=h_{2 \alpha+3 \beta}\left(z^{-1}\right) \chi_{2 \alpha+3 \beta}(-z) k_{2 \alpha+3 \beta}(z)$, and then $h_{2 \alpha+3 \beta}\left(z^{-1}\right)=h\left(z^{-1}, 1\right), h_{2 \alpha+3 \beta}(z) \cdot n^{-}(x, y)=n^{-}\left(z^{-1} x, z^{-1} y\right)$, and $f_{s}\left(n^{-}(x, y) \chi_{2 \alpha+3 \beta}(-z)\right)=f_{s}\left(n^{-}\left(x+z y^{3}, y\right)\right)$. Hence we obtain

$$
\begin{aligned}
& I_{---}=\int_{F^{2} ;|z|,|u|,|v|>1} f_{s}\left(n^{-}(x, y)\right)|v|^{-3 s+1}|u|^{-9 s+4}|z|^{-6 s+2} \\
& \cdot \psi_{\circ}\left(a v-v u^{3}+\left(x z-z^{2} y^{3}-u^{3} z^{2}-3 u y z^{2}(y+u)\right) v^{-1}-u^{2} z(2 u+3 y)\right) d n .
\end{aligned}
$$

Following the same procedure, we obtain the following 16 integrals

$$
I_{++++}=\int_{F} f_{s}\left(n^{-}(x)\right) \psi_{\circ}(x) d x
$$




$$
\begin{aligned}
& I_{+++-}=\int_{F ;|y|>1} f_{s}\left(n^{-}(x)\right)|y|^{-9 s+3} \psi_{\circ}\left(x y^{3}\right) d x d y \\
& I_{++-+}=\int_{F ;|y| \leq 1,|z|>1} f_{s}\left(n^{-}(x)\right)|z|^{-6 s+2} \psi_{\circ}\left(x z-z^{2} y^{3}\right) d n \\
& I_{++--}=\int_{F ;|z|,|y|>1} f_{s}\left(n^{-}(x)\right)|y|^{-9 s+3}|z|^{-6 s+2} \psi_{\circ}\left(x z y^{3}-z^{2} y^{3}\right) d n, \\
& I_{+-++}=\int_{F ;|u|>1 ;|z|,|y| \leq 1} f_{s}\left(n^{-}(x)\right)|u|^{-9 s+4} \psi_{\circ}\left(x-3 u y^{2}-3 u^{2} y z-u^{3} z^{2}\right) d n \\
& I_{+-+-}=\int_{F ;|u|,|y|>1 ;|z| \leq 1} f_{s}\left(n^{-}(x)\right)|y|^{-9 s+3}|u|^{-9 s+4} \\
& \cdot \psi_{\circ}\left(x y^{3}-3 u y(y+u z)-u^{3} z^{2}\right) d n \\
& I_{+--+}=\int_{F ;|u|,|z|>1 ;|y| \leq 1} f_{s}\left(n^{-}(x)\right)|u|^{-9 s+4}|z|^{-6 s+2} \\
& \cdot \psi_{\circ}\left(z x-z^{2} y^{3}-3 u y^{2} z^{2}-3 u^{2} y z^{2}-u^{3} z^{2}\right) d n \\
& I_{+---}=\int_{F ;|u|,|z|,|y|>1} f_{s}\left(n^{-}(x)\right)|u|^{-9 s+4}|z|^{-6 s+2}|y|^{-9 s+3} \\
& \cdot \psi_{\circ}\left(x y^{3} z-z^{2} y^{3}-3 u y^{2} z^{2}-3 u^{2} y z^{2}-u^{3} z^{2}\right) d n \\
& I_{-+++}=\int_{F ;|v|>1 ;|u| \leq 1} f_{s}\left(n^{-}(x)\right)|v|^{-3 s+1} v \psi_{\circ}\left(a v-u^{3} v+x v^{-1}\right) d n \\
& I_{-++-}=\int_{F ;|v|,|y|>1 ;|u| \leq 1} f_{s}\left(n^{-}(x)\right)|v|^{-3 s+1}|y|^{-9 s+3} \\
& \cdot \psi_{\circ}\left(a v-u^{3} v+x y^{3} v^{-1}-3 u y\right) d n \\
& I_{-+-+}=\int_{F ;|v|,|z|>1 ;|u|,|y| \leq 1} f_{s}\left(n^{-}(x)\right)|v|^{-3 s+1}|z|^{-6 s+2} \\
& \cdot \psi_{\circ}\left(a v-u^{3} v+x z v^{-1}-z^{2} y^{3} v^{-1}+z-3 u y z\right) d n \\
& I_{-+--}=\int_{F ;|v|,|z|,|y|>1 ;|u| \leq 1} f_{s}\left(n^{-}(x)\right)|v|^{-3 s+1}|z|^{-6 s+2}|y|^{-9 s+3} \\
& \cdot \psi_{\circ}\left(a v-u^{3} v+x y^{3} z v^{-1}-z^{2} y^{3} v^{-1}+z-3 u y z\right) d n \\
& I_{--++}=\int_{F ;|v|,|u|>1 ;|z|,|y| \leq 1} f_{s}\left(n^{-}(x)\right)|v|^{-3 s+1}|u|^{-9 s+4} \\
& \cdot \psi_{\circ}\left(a v-u^{3} v+\left(x-3 u y(y+u z)-u^{3} z^{2}\right) v^{-1}-3 u^{2} y-2 u^{3} z\right) d n \\
& I_{--+-}=\int_{F ;|v|,|u|,|y|>1 ;|z| \leq 1} f_{s}\left(n^{-}(x)\right)|v|^{-3 s+1}|u|^{-9 s+4}|y|^{-9 s+3} \\
& \cdot \psi_{\circ}\left(a v-u^{3} v+\left(x y^{3}-3 u y(y+u z)-u^{3} z^{2}\right) v^{-1}-3 u^{2} y-2 u^{3} z\right) d n \\
& I_{---+}=\int_{F ;|v|,|u|,|z|>1 ;|y| \leq 1} f_{s}\left(n^{-}(x)\right)|v|^{-3 s+1}|u|^{-9 s+4}|z|^{-6 s+2} \\
& \cdot \psi_{\circ}\left(a v-u^{3} v+\left(x z-z^{2} y^{3}-3 u y z^{2}(y+u)-u^{3} z^{2}\right) v^{-1}-(3 y+2 u) z u^{2}\right) d n
\end{aligned}
$$




$$
\begin{aligned}
& I_{----}=\int_{F ;|v|,|u|,|z|,|y|>1} f_{s}\left(n^{-}(x)\right)|v|^{-3 s+1}|u|^{-9 s+4}|z|^{-6 s+2}|y|^{-9 s+3} \\
& \cdot \psi_{\circ}\left(a v-u^{3} v+\left(x y^{3} z-z^{2} y^{3}-3 u y z^{2}(y+u)-u^{3} z^{2}\right) v^{-1}-(3 y+2 u) z u^{2}\right) d n .
\end{aligned}
$$

Proposition 1. Assume that a is a unit in F. We have

$$
I_{+++-}=I_{++-+}=I_{++--}=I_{+-+-}=I_{+--+}=I_{+---}=0
$$

and

$$
I_{-++-}=I_{-+--}=I_{--++}=I_{--+-}=I_{----}=0 .
$$

Proposition 2. Assume that $a$ is a unit in $F$. We obtain that

$$
\begin{aligned}
& I_{++++}=\left(1-q^{-3 s}\right) \\
& I_{+-++}=-\left(1-q^{-3 s}\right)+\left(1-q^{-3 s}\right)\left(1-q^{-9 s+2}\right)\left(1-q^{-9 s+3}\right)^{-1} .
\end{aligned}
$$

Proposition 1 and 2 imply that

Corollary 1. Assume that $a$ is a unit in F. We have

$$
I_{+}=\left(1-q^{-3 s}\right)\left(1-q^{-9 s+2}\right)\left(1-q^{-9 s+3}\right)^{-1} .
$$

Proposition 3. Assume that $a$ is a unit in $F$.

$$
I_{-+++}= \begin{cases}\left(1-q^{-3 s}\right)\left(-q^{-3 s+1}-q^{-6 s+2}\right), & \text { if } a \notin \mathcal{O}^{\times, 3} \\ \left(1-q^{-3 s}\right)\left(2 q^{-3 s+1}+2 q^{-6 s+2}\right), & \text { if } a \in \mathcal{O}^{\times, 3} .\end{cases}
$$

The remainder are two cases $I_{-+-+}$and $I_{---+}$, which need more computation. First we are going to prove the following Lemma, which will be applied to the calculation of the integrals $I_{-+-+}$and $I_{---+}$.

Lemma 3. Let $b$ be an element of $F^{\times}$. Then

$$
\int_{|x|=1} \psi_{\circ}\left(b \pi^{m} x^{3}\right) d x= \begin{cases}0, & \text { if }\left|b \pi^{m+1}\right|>1, \\ q^{-1} \sum_{\chi \in \mathbb{F}_{q}^{\times}} \psi_{\circ}\left(b \pi^{m} \chi^{3}\right), & \text { if }\left|b \pi^{m+1}\right| \leq 1 .\end{cases}
$$

Proof. Let $x=\chi+\pi x_{1}$ with $\chi \in \mathbb{F}_{q}^{\times}$and $x_{1} \in \mathcal{O}$. Then

$$
\begin{aligned}
B(b, m) & =\int_{|x|=1} \psi_{\circ}\left(b \pi^{m} x^{3}\right) d x \\
& =q^{-1} \sum_{\chi \in \mathbb{F}_{q}^{\times}} \psi_{\circ}\left(b \pi^{m} \chi^{3}\right) \int_{\left|x_{1}\right| \leq 1} \psi_{\circ}\left(b \pi^{m+1}\left[3 \chi^{2}+3 \chi \pi x_{1}+\pi^{2} x_{1}^{2}\right] x_{1}\right) d x_{1} .
\end{aligned}
$$


By the key lemma in [Igu], the polynomial map

$$
x_{1} \mapsto\left[3 \chi^{2}+3 \chi \pi x_{1}+\pi^{2} x_{1}^{2}\right] x_{1}
$$

is a measure-preserved analytic homeomorphism from $\mathcal{O}$ to itself since we assume that $3 \chi^{2}$ is a unit in $\mathcal{O}$. Hence, by substituting the polynomial, we obtain

$$
\begin{aligned}
B(b, m) & =q^{-1} \sum_{\chi \in \mathbb{F}_{q}^{\times}} \psi_{\circ}\left(b \pi^{m} \chi^{3}\right) \int_{\left|x_{2}\right| \leq 1} \psi_{\circ}\left(b \pi^{m+1} x_{2}\right) d x_{2} \\
& = \begin{cases}0, & \text { if }\left|b \pi^{m+1}\right|>1, \\
q^{-1} \sum_{\chi \in \mathbb{F}_{q}^{\times}} \psi_{\circ}\left(b \pi^{m} \chi^{3}\right), & \text { if }\left|b \pi^{m+1}\right| \leq 1 .\end{cases}
\end{aligned}
$$

Proposition 4. Assume that $F$ contains the third root of unity and $|a|=$ 1. Then we have

$$
I_{-+-+}= \begin{cases}\left(1-q^{-3 s}\right) q^{-9 s+2}\left[1+q^{-1} S(a)\right], & \text { if } a \notin \mathcal{O}^{\times, 3} \\ \left(1-q^{-3 s}\right) q^{-9 s+2}\left[-2+q^{-1} S(a)\right], & \text { if } a \in \mathcal{O}^{\times, 3}\end{cases}
$$

where

$$
S(a):=\sum_{\xi, \zeta, \nu \in \mathbb{F}_{q}^{\times} ; \mu \in \mathbb{F}_{q}} \psi_{\circ}\left(\left[a \xi^{3}-\mu^{3}-\zeta^{2}+\zeta-3 \mu \zeta\right] \pi^{-1} \nu\right)
$$

is the exponential sum as in Lemma 2.

Proof. By the integration with respect to the variable $x$, we obtain

$$
\begin{aligned}
I_{-+-+}= & \frac{\left(1-q^{-3 s}\right)}{\left(1-q^{-3 s+1}\right)} \int_{|v|,|z|>1 ;|u|,|y|,\left|z v^{-1}\right| \leq 1}\left(1-q^{-3 s+1}\left|z v^{-1}\right|^{3 s-1}\right) \\
& \cdot|v|^{-3 s+1}|z|^{-6 s+2} \psi_{\circ}\left(a v-u^{3} v-z^{2} y^{3} v^{-1}+z-3 u y z\right) d n .
\end{aligned}
$$

By substitution, $z v^{-1} \mapsto z$, we have

$$
\begin{gathered}
I_{-+-+}=\frac{\left(1-q^{-3 s}\right)}{\left(1-q^{-3 s+1}\right)} \int_{|v|>\left|z^{-1}\right| ;|u|,|y|,|z| \leq 1}\left(1-q^{-3 s+1}|z|^{3 s-1}\right)|v|^{-9 s+4}|z|^{-6 s+2} \\
\cdot \psi_{\circ}\left(\left[a-u^{3}-z^{2} y^{3}+z-3 u y z\right] v\right) d n .
\end{gathered}
$$

Then, making substitutions: $z y^{3} \mapsto z, v y^{-3} \mapsto v, u y \mapsto u$, and $z y^{3} \mapsto z$, we obtain

$$
I_{-+-+}=\frac{\left(1-q^{-3 s}\right)}{\left(1-q^{-3 s+1}\right)} \int_{|v|>\left|z^{-1}\right| ;|,| z|\leq| y^{3}|;| u|\leq| y \mid \leq 1}\left(1-q^{-3 s+1}|z|^{3 s-1}|y|^{-9 s+3}\right)
$$




$$
\cdot|v|^{-9 s+4}|y|^{-9 s+5}|z|^{-6 s+2} \psi_{\circ}\left(\left[a y^{3}-u^{3}-z^{2}+z-3 u z\right] v\right) d n .
$$

Let $y=\pi^{i} y_{1}$ for $i=0,1,2, \cdots$. Then

$$
\begin{gathered}
I_{-+-+}=\frac{\left(1-q^{-3 s}\right)}{\left(1-q^{-3 s+1}\right)} \sum_{i=0}^{\infty} q^{i(9 s-6)} \int_{|v|>\left|z^{-1}\right| ;,|z| \leq q^{-3 i} ;|u| \leq q^{-i}}\left(1-q^{-3 s+1+i(9 s-3)}|z|^{3 s-1}\right) \\
\cdot|v|^{-9 s+4}|z|^{-6 s+2} \psi_{\circ}\left(\left[-u^{3}-z^{2}+z-3 u z\right] v\right) \int_{\left|y_{1}\right|=1} \psi_{\circ}\left(a v \pi^{3 i} y_{1}^{3}\right) d y_{1} d *
\end{gathered}
$$

where $d *=d u d v d z$. By Lemma 3 , the integration in $y_{1}$ can be simply expressed as

$$
\int_{\left|y_{1}\right|=1} \psi_{\circ}\left(a v \pi^{3 i} y_{1}^{3}\right) d y_{1}= \begin{cases}0, & \text { if }|v| \geq q^{3 i+2} \\ q^{-1} \sum_{\xi \in \mathbb{F}_{q}^{\times}} \psi_{\circ}\left(a v \pi^{3 i} \xi^{3}\right), & \text { if }|v|=q^{3 i+1}\end{cases}
$$

Since $|v|>\left|z^{-1}\right| \geq q^{3 i}$, the integral $I_{-+-+}$does not vanish only if $|v|=q^{3 i+1}$. This implies $|z|=q^{-3 i}$.

By setting $u \mapsto \pi^{i} u, z \mapsto \pi^{3 i} z$, and $v \mapsto \pi^{-3 i-1} v$, we get

$$
\begin{aligned}
I_{-+-+}= & \left(1-q^{-3 s}\right) q^{-9 s+4} \sum_{i=0}^{\infty} q^{-i} \sum_{\xi \in \mathbb{F}_{q}^{\times}} \\
& \int_{|u| \leq 1 ;|z|=|v|=1} \psi_{\circ}\left(\left[a \xi^{3}-u^{3}-z^{2} \pi^{3 i}+z-3 u z \pi^{i}\right] \pi^{-1} v\right) d v d z d u \\
= & \left(1-q^{-3 s}\right) q^{-9 s+4} \sum_{i=0}^{\infty} q^{-i} \sum_{\xi \in \mathbb{F}_{q}^{\times}} J(i, \xi) .
\end{aligned}
$$

The inner integral $J(i, \xi)$ will be treated separately for $i \geq 1$ and for $i=0$.

When $i \geq 1$, one has

$$
J(i \geq 1, \xi)=\int_{|u| \leq 1 ;|z|=|v|=1} \psi_{\circ}\left(\left[a \xi^{3}-u^{3}+z\right] \pi^{-1} v\right) d v d z d u,
$$

since the additive character $\psi_{\circ}$ is unramified. Then we have

$$
\begin{aligned}
J(i \geq 1, \xi) & =-q^{-1} \int_{|u| \leq 1 ;|v|=1} \psi_{\circ}\left(\left[a \xi^{3}-u^{3}\right] \pi^{-1} v\right) d v d u \\
& =-q^{-1} \int_{|u| \leq 1 ;|v|=1} \psi_{\circ}\left(\left[a-u^{3}\right] \pi^{-1} v\right) d v d u \\
& =-q^{-2} \sum_{\mu \in \mathbb{F}_{q}} \int_{|v|=1} \psi_{\circ}\left(\left[a-\mu^{3}\right] \pi^{-1} v\right) d v d z d u \\
& = \begin{cases}q^{-2}, & \text { if } a \notin \mathcal{O}^{\times, 3} ; \\
-2 q^{-2}, & \text { if } a \in \mathcal{O}^{\times, 3} .\end{cases}
\end{aligned}
$$


Hence we have that

$$
\sum_{i=1}^{\infty} q^{-i} \sum_{\xi \in \mathbb{F}_{q}^{\times}} J(i, \xi)= \begin{cases}q^{-2}, & \text { if } a \notin \mathcal{O}^{\times, 3} \\ -2 q^{-2}, & \text { if } \quad a \in \mathcal{O}^{\times, 3}\end{cases}
$$

When $i=0$, we have

$$
\begin{aligned}
J(0, \xi) & =\int_{|u| \leq 1 ;|z|=|v|=1} \psi_{\circ}\left(\left[a \xi^{3}-u^{3}-z^{2}+z-3 u z\right] \pi^{-1} v\right) d v d z d u \\
& =q^{-3} \sum_{\zeta, \nu \in \mathbb{F}_{q}^{\times} ; \mu \in \mathbb{F}_{q}} \psi_{\circ}\left(\left[a \xi^{3}-\mu^{3}-\zeta^{2}+\zeta-3 \mu \zeta\right] \pi^{-1} \nu\right) .
\end{aligned}
$$

Hence $\sum_{\xi \in \mathbb{F}_{q}^{\times}} J(0, \xi)=q^{-3} S(a)$. Therefore we obtain

$$
I_{-+-+}= \begin{cases}\left(1-q^{-3 s}\right) q^{-9 s+2}\left[1+q^{-1} S(a)\right], & \text { if } a \notin \mathcal{O}^{\times, 3} \\ \left(1-q^{-3 s}\right) q^{-9 s+2}\left[-2+q^{-1} S(a)\right], & \text { if } a \in \mathcal{O}^{\times, 3}\end{cases}
$$

This proves the proposition.

Proposition 5. Assume that $F$ contains the third root of unity and $|a|=$ 1. Then we have

$$
I_{---+}=\left(1-q^{-3 s}\right)\left(q^{-18 s+5}-q^{-18 s+6}\right)\left(1-q^{-9 s+3}\right)^{-1} .
$$

Proof. First integrating variable $x$, we have

$$
\begin{aligned}
I_{---+}= & \frac{\left(1-q^{-3 s}\right)}{\left(1-q^{-3 s+1}\right)} \int_{|v|,|u|,|z|>1 ;|y|,\left|z v^{-1}\right| \leq 1}\left(1-q^{-3 s+1}\left|z v^{-1}\right|^{3 s-1}\right) \\
& \cdot|v|^{-3 s+1}|u|^{-9 s+4}|z|^{-6 s+2} \psi_{\circ}\left(a v-u^{3} v\right. \\
& \left.\quad-\left(z^{2} y^{3}+3 u y z^{2}(y+u)+u^{3} z^{2}\right) v^{-1}-(3 y+2 u) z u^{2}\right) d n .
\end{aligned}
$$

By substituting $z v^{-1} \mapsto z$, the integral is reduced to be

$$
\begin{aligned}
I_{---+}= & \frac{\left(1-q^{-3 s}\right)}{\left(1-q^{-3 s+1}\right)} \int_{|v|,|u|,|z v|>1 ;|y|,|z| \leq 1}\left(1-q^{-3 s+1}|z|^{3 s-1}\right)|v|^{-9 s+4}|u|^{-9 s+4} \\
& \cdot|z|^{-6 s+2} \psi_{\circ}\left(\left[a-z^{2} y^{3}-3 u y^{2} z^{2}-3 u^{2} z(z+1) y-(z+1)^{2} u^{3}\right] v\right) d n .
\end{aligned}
$$

It follows that if $|z+1|=1$, then the integral will be zero by Lemma 1 . Hence we only need to consider the condition $|z+1|<1$, i.e. $z \in-1+\pi \mathcal{O}$, and the integral must be

$$
I_{---+}=\left(1-q^{-3 s}\right) \int_{|v|,|u|>1 ;|y| \leq 1 ;|z+1|<1}|v|^{-9 s+4}|u|^{-9 s+4}
$$




$$
\cdot \psi_{\circ}\left(\left[a-z^{2} y^{3}-3 u y^{2} z^{2}-3 u^{2} z(z+1) y-(z+1)^{2} u^{3}\right] v\right) d n .
$$

Making the following substitutions: $y u^{-1} \mapsto y, v u^{3} \mapsto v, u^{-1} \mapsto u, y z \mapsto y$, and then $z+1 \mapsto z$, we deduce the integral to be

$$
\begin{aligned}
J=\int_{\left|v u^{3}\right|>1 ;|u|,|z|<1 ;|y| \leq|u|}|v|^{-9 s+4}|u|^{-18 s+8} \\
\cdot \psi_{\circ}\left(\left[a v u^{3}+(z-1)^{-1} y^{3}-3 y^{2}-3 z y-z^{2}\right] v\right) d n
\end{aligned}
$$

where $I_{---+}=\left(1-q^{-3 s}\right) J$. Let $h(y, z)=-(z-1)^{-1} y^{3}-3 y^{2}-3 z y-z^{2}$. Setting $u \mapsto \pi^{i} u$, we obtain

$$
\begin{aligned}
& J=\sum_{i=1}^{\infty} q^{i(18 s-9)} \int_{|y| \leq q^{-i} ;|v| \geq q^{3 i+1} ;|z|<1}|v|^{-9 s+4} \\
& \cdot \psi_{\circ}(h(y, z) v) \int_{|u|=1} \psi_{\circ}\left(a v \pi^{3 i} u^{3}\right) d u d *
\end{aligned}
$$

where $d *=d v d y d z$. By Lemma 3, the inner integration about variable $u$ can be deduced as follows:

$$
\int_{|u|=1} \psi_{\circ}\left(a v \pi^{3 i} u^{3}\right) d u= \begin{cases}0, & \text { if }\left|v \pi^{3 i}\right| \geq q^{2}, \\ q^{-1} \sum_{\mu \in \mathbb{F}_{q}^{\times}} \psi_{\circ}\left(a v \pi^{3 i} \mu^{3}\right), & \text { if }\left|v \pi^{3 i}\right|=q .\end{cases}
$$

Plugging this into the original integral and setting $v \mapsto \pi^{-3 i-1} v$, we get

$$
\begin{aligned}
J= & q^{-9 s+4} \sum_{i=1}^{\infty} q^{i(-9 s+6)} \\
& \cdot \sum_{\mu \in \mathbb{F}_{q}^{\times}} \int_{|y| \leq q^{-i} ;|v|=1 ;|z|<1} \psi_{\circ}\left(\left[h(y, z) \pi^{-3 i-1}+a \pi^{-1} \mu^{3}\right] v\right) d v d y d z \\
= & q^{-9 s+4} \sum_{i=1}^{\infty} q^{i(-9 s+6)} J(i),
\end{aligned}
$$

and

$$
\begin{aligned}
J(i)= & \sum_{\mu \in \mathbb{F}_{q}^{\times}} \int_{|y| \leq q^{-i} ;|v|=1 ;|z|<1} \psi_{\circ}\left(\left[h(y, z) \pi^{-3 i-1}+a \pi^{-1} \mu^{3}\right] v\right) d v d y d z \\
= & \sum_{\nu, \mu \in \mathbb{F}_{q}^{\times}} \psi_{\circ}\left(a \pi^{-1} \mu^{3} \nu\right) \int_{|y| \leq q^{-i} ;|z|<1} \psi_{\circ}\left(h(y, z) \pi^{-3 i-1} \nu\right) \\
& \cdot \int_{|v|<1} \psi_{\circ}\left(\left[3 y^{2}+3 y z+z^{2}\right] \pi^{-3 i-1} v\right) d v d y d z .
\end{aligned}
$$


The nonvanishing of the integration about $v$ implies $|z| \leq q^{-i}$. Then we write

$$
J(i)=\sum_{\nu, \mu \in \mathbb{F}_{q}^{\times}} \psi_{\circ}\left(a \pi^{-1} \mu^{3} \nu\right)\left[J_{1}(i, \nu)+J_{2}(i, \nu)\right],
$$

where

$$
\begin{aligned}
J_{1}(i, \nu)= & \int_{|y|,|z| \leq q^{-i-1}} \psi_{\circ}\left(h(y, z) \pi^{-3 i-1} \nu\right) \\
& \cdot \int_{|v|<1} \psi_{\circ}\left(\left[3 y^{2}+3 y z+z^{2}\right] \pi^{-3 i-1} v\right) d v d y d z
\end{aligned}
$$

and

$$
\begin{aligned}
J_{2}(i, \nu)= & \int_{|y|,|z|=q^{-i}} \psi_{\circ}\left(h(y, z) \pi^{-3 i-1} \nu\right) \\
& \cdot \int_{|v|<1} \psi_{\circ}\left(\left[3 y^{2}+3 y z+z^{2}\right] \pi^{-3 i-1} v\right) d v d y d z .
\end{aligned}
$$

First we are going to calculate the integral $J_{1}(i, \nu)$. We make substitutions: $y \mapsto \pi^{i+1} y, z \mapsto \pi^{i+1} z$, and $v \mapsto \pi v$, and obtain

$$
\begin{aligned}
J_{1}(i, \nu)= & q^{-(2 i+3)} \int_{|y|,|z| \leq 1} \psi_{\circ}\left(-\left(\pi^{i+1} z-1\right)^{-1} y^{3} \pi^{2} \nu-\left[3 y^{2}+3 y z+z^{2}\right] \pi^{1-i} \nu\right) \\
& \cdot \int_{|v| \leq 1} \psi_{\circ}\left(\left[3 y^{2}+3 y z+z^{2}\right] \pi^{2-i} v\right) d v d y d z \\
= & q^{-(2 i+3)} \int_{|y|,|z| \leq 1 ;\left|3 y^{2}+3 y z+z^{2}\right| \leq q^{2-i}} \psi_{\circ}\left(-\left[3 y^{2}+3 y z+z^{2}\right] \pi^{1-i} \nu\right) d y d z .
\end{aligned}
$$

Since $3 y^{2}+3 y z+z^{2}=(z-(\omega-1) y)\left(z-\left(\omega^{2}-1\right) y\right)$ holds over $\mathcal{O}$, we set

$$
\left\{\begin{array}{l}
z_{1}=z-(\omega-1) y \\
y_{1}=z-\left(\omega^{2}-1\right) y .
\end{array}\right.
$$

Then the Jacobi of this transform is $\omega-\omega^{2}$, which is a unit in $\mathcal{O}$. This means that the transform is by the key lemma in [Igu] a measure-preserved analytic homeomorphism from $\mathcal{O}^{2}$ to $\mathcal{O}^{2}$. Hence the integral can be written as

$$
J_{1}(i, \nu)=q^{-(2 i+3)} \int_{|y|,|z| \leq 1 ;|y z| \leq q^{2-i}} \psi_{\circ}\left(-y z \pi^{1-i} \nu\right) d y d z=q^{-(2 i+3)} D(i, \nu) .
$$

It is easy to verify that when $i=1$, the integral

$$
D(1, \nu)=\int_{|y|,|z| \leq 1} \psi_{\circ}\left(-y z \pi^{0} \nu\right) d y d z=1,
$$


and when $i=2$, the integral

$$
D(2, \nu)=\int_{|y|,|z| \leq 1} \psi_{\circ}\left(-y z \pi^{-1} \nu\right) d y d z=q^{-1} .
$$

For $i \geq 3$, the integral can be reduced as follows.

$$
\begin{aligned}
D(i \geq 3, \nu)= & \int_{\left|z_{1}\right| \leq 1 ;\left|y_{1}\right| \leq \min \left\{q^{2-i}, 1\right\}} \psi_{\circ}\left(-y_{1} z_{1} \pi^{1-i} \nu\right) d y_{1} d z_{1} \\
& \quad+q^{-1} \int_{\left|y_{1}\right|,\left|z_{1}\right| \leq 1 ;\left|y_{1} z_{1}\right| \leq q^{3-i}} \psi_{\circ}\left(-y_{1} z_{1} \pi^{2-i} \nu\right) d y_{1} d z_{1} \\
= & q^{-1} \int_{\left|y_{1}\right|,\left|z_{1}\right| \leq 1 ;\left|y_{1} z_{1}\right| \leq q^{3-i}} \psi_{\circ}\left(-y_{1} z_{1} \pi^{2-i} \nu\right) d y_{1} d z_{1} \\
= & \cdots \\
= & q^{-(i-2)} \int_{\left|y_{1}\right|,\left|z_{1}\right| \leq 1 ;\left|y_{1} z_{1}\right| \leq q^{0}} \psi_{\circ}\left(-y_{1} z_{1} \pi^{-1} \nu\right) d y_{1} d z_{1} \\
= & q^{-i+1} .
\end{aligned}
$$

Hence we obtain that $J_{1}(i, \nu)=q^{-(3 i+2)}$, which is independent of the variable $\nu$, and then that

$$
\begin{aligned}
J(i) & =\sum_{\nu, \mu \in \mathbb{F}_{q}^{\times}} \psi_{\circ}\left(a \pi^{-1} \mu^{3} \nu\right)\left[q^{-(3 i+2)}+J_{2}(i, \nu)\right] \\
& =-(q-1) q^{-(3 i+2)}+\sum_{\nu, \mu \in \mathbb{F}_{q}^{\times}} \psi_{\circ}\left(a \pi^{-1} \mu^{3} \nu\right) J_{2}(i, \nu) .
\end{aligned}
$$

Next we are going to show that

$$
\sum_{\nu, \mu \in \mathbb{F}_{q}^{\times}} \psi_{\circ}\left(a \pi^{-1} \mu^{3} \nu\right) J_{2}(i, \nu)=0 .
$$

We notice first of all that

$$
\int_{|v|<1} \psi_{\circ}\left(\left[3 y^{2}+3 y z+z^{2}\right] \pi^{-3 i-1} v\right) d v= \begin{cases}0, & \text { if }\left|3 y^{2}+3 y z+z^{2}\right|>q^{-3 i} \\ q^{-1}, & \text { if }\left|3 y^{2}+3 y z+z^{2}\right| \leq q^{-3 i}\end{cases}
$$

After setting $y \mapsto \pi^{i} y$ and $z \mapsto \pi^{i} z$, we obtain

$$
\begin{aligned}
J_{2}(i, \nu)= & q^{-2 i-1} \int_{|y|=|z|=1 ;\left|3 y^{2}+3 y z+z^{2}\right| \leq q^{-i}} \psi_{\circ} \\
& \cdot\left(\frac{-y^{3} \nu}{\left(z \pi^{i}-1\right) \pi}-\left(3 y^{2}+3 y z+z^{2}\right) \pi^{-i-1} \nu\right) d y d z .
\end{aligned}
$$

Since $\left(z \pi^{i}-1\right)$ is a unit in $F$ for $i \geq 1$, by setting $\nu\left(z \pi^{i}-1\right)^{-1} \mapsto \nu$,

$$
\sum_{\nu, \mu \in \mathbb{F}_{q}^{\times}} \psi_{\circ}\left(a \pi^{-1} \mu^{3} \nu\right) J_{2}(i, \nu)
$$




$$
\begin{aligned}
& =\sum_{\nu, \mu \in \mathbb{F}_{q}^{\times}} \psi_{\circ}\left(-a \pi^{-1} \mu^{3} \nu\right) \\
& \cdot \int_{|y|=|z|=1 ;\left|3 y^{2}+3 y z+z^{2}\right| \leq q^{-i}} \psi_{\circ}\left(-y^{3} \pi^{-1} \nu+\left(3 y^{2}+3 y z+z^{2}\right) \pi^{-i-1} \nu\right) d y d z .
\end{aligned}
$$

It suffices to show that the integral

$$
\int_{|y|=|z|=1 ;\left|3 y^{2}+3 y z+z^{2}\right| \leq q^{-i}} \psi_{\circ}\left(-y^{3} \pi^{-1} \nu+\left(3 y^{2}+3 y z+z^{2}\right) \pi^{-i-1} \nu\right) d y d z,
$$

which is denoted by $J_{2}^{\prime}(i, \nu)$, is equal zero. Since we assume that $F$ contains the third root of unity $\omega$, we can decompose the quadratic polynomial as

$$
3 y^{2}+3 y z+z^{2}=(z-(\omega-1) y)\left(z-\left(\omega^{2}-1\right) y\right) \in \pi^{i} \mathcal{O} .
$$

Let $z_{1}=z-(\omega-1) y$. Then $z-\left(\omega^{2}-1\right) y=z_{1}+\left(\omega-\omega^{2}\right) y$. Since $\left(\omega-\omega^{2}\right) y$ is a unit, one knows that

$$
z_{1}\left(z_{1}+\left(\omega-\omega^{2}\right) y\right) \in \pi^{i} \mathcal{O}
$$

implies that $z_{1} \in \pi^{i} \mathcal{O}$ or $z_{1}+\left(\omega-\omega^{2}\right) y \in \pi^{i} \mathcal{O}$. Without lose of generality, we may assume that $z_{1} \in \pi^{i} \mathcal{O}$. By replacing the part related to $z$, we obtain

$$
\begin{aligned}
J_{2}^{\prime}(i, \nu) & =q^{-2 i-1} \int_{|y|=1 ;\left|z_{1}\right| \leq q^{-i}} \psi_{\circ}\left(-y^{3} \pi^{-1} \nu+z_{1}\left(z_{1}+\left(\omega-\omega^{2}\right) y\right) \pi^{-i-1} \nu\right) d y d z_{1} \\
& =q^{-2 i-1} \int_{|y|=1 ;\left|z_{1}\right| \leq q^{-i}} \psi_{\circ}\left(-y^{3} \pi^{-1} \nu+z_{1}\left(\omega-\omega^{2}\right) y \pi^{-i-1} \nu\right) d y d z_{1} \\
& =0
\end{aligned}
$$

since $i \geq 1$ and the integration with respect to $z_{1}$ is zero. In other words, we have

$$
J(i)=-(q-1) q^{-(3 i+2)} .
$$

Finally we obtain

$$
\begin{aligned}
J=q^{-9 s+4} \sum_{i=1}^{\infty} q^{i(-9 s+6)} J(i) & =-(q-1) q^{-9 s+2} \sum_{i=1}^{\infty} q^{i(-9 s+3)} \\
& =\frac{-\left(1-q^{-1}\right) q^{-18 s+6}}{\left(1-q^{-9 s+3}\right)}
\end{aligned}
$$

This finishes the proof.

Summarizing the results from the computations above, we obtain:

Corollary 2. Assume that the local field contains the third root of unity and $a$ be a unit in $F$. For the unramified additive character $\psi_{\sigma_{3}}$ associated to $\sigma_{3}=(1,0,0, a)$, we have,

(1) when $a \in \mathcal{O}^{\times, 3}$,

$$
I\left(f_{s}, \psi_{\sigma_{3}}\right)=\left(1-q^{-3 s}\right)\left(1+q^{-3 s+1}\right)\left(1+q^{-3 s+1}+q^{-6 s+2}\right)
$$




$$
+\frac{\left(1-q^{-3 s}\right)\left(q^{-9 s+1}-q^{-18 s+4}\right)(S(a)-3 q)}{\left(1-q^{-9 s+3}\right)}
$$

and

(2) when $a \notin \mathcal{O}^{\times, 3}$,

$$
\begin{aligned}
I\left(f_{s}, \psi_{\sigma_{3}}\right)=\left(1-q^{-3 s}\right)\left(1-q^{-3 s+1}\right)\left(1-q^{-6 s+2}\right) \\
+\frac{\left(1-q^{-3 s}\right)\left(q^{-9 s+1}-q^{-18 s+4}\right) S(a)}{\left(1-q^{-9 s+3}\right)} .
\end{aligned}
$$

Proof. Note that $I\left(f_{s}, \psi_{\sigma_{3}}\right)=I_{+}+I_{-}$. By Corollary 1, we have

$$
I_{+}=\left(1-q^{-3 s}\right)\left(1-q^{-9 s+2}\right)\left(1-q^{-9 s+3}\right)^{-1} .
$$

By Proposition 3, 4, and 5, we have, when $a \in \mathcal{O}^{\times, 3}$,

$$
\begin{gathered}
I_{-}=\left(1-q^{-3 s}\right)\left(1+q^{-3 s+1}\right)\left(1+q^{-3 s+1}+q^{-6 s+2}\right)-I_{+} \\
+\frac{\left(1-q^{-3 s}\right)\left(q^{-9 s+1}-q^{-18 s+4}\right)(S(a)-3 q)}{\left(1-q^{-9 s+3}\right)},
\end{gathered}
$$

and when $a \notin \mathcal{O}^{\times, 3}$,

$$
\begin{gathered}
I_{-}=\left(1-q^{-3 s}\right)\left(1-q^{-3 s+1}\right)\left(1-q^{-6 s+2}\right)-I_{+} \\
+\frac{\left(1-q^{-3 s}\right)\left(q^{-9 s+1}-q^{-18 s+4}\right) S(a)}{\left(1-q^{-9 s+3}\right)} .
\end{gathered}
$$

The proposition follows.

5.2. Case: $\sigma_{1}=(0,1,1,0)$. In this case, the integral is

$$
I=\int_{F^{5}} f_{s}\left(n^{-}(x, y,-z,-u,-v)\right) \psi_{\circ}(u+y) d n .
$$

Using the same notations, we deduce easily that $I_{--}=I_{+-}=0$ and

$$
\begin{aligned}
I= & \int_{F^{3}} f_{s}\left(n^{-}(x, y,-z)\right) \psi_{\sigma_{1}}(y) d z d x d y \\
& \cdot\left(1+\int_{|u| \leq 1 ;|v|>1}|v|^{-3 s+1} \psi_{\sigma_{1}}\left(\left(u+u^{2}\right) v\right) d v d u\right) .
\end{aligned}
$$

The straightforward calculation gives us

$$
\int_{F^{3}} f_{s}\left(n^{-}(x, y,-z)\right) \psi_{\sigma_{1}}(y) d z d x d y=\frac{\left(1-q^{-3 s}\right)\left(1-q^{-9 s+3}\right)}{\left(1-q^{-3 s+1}\right)}
$$


and

$$
1+\int_{|u| \leq 1 ;|v|>1}|v|^{-3 s+1} \psi_{\sigma_{1}}\left(\left(u+u^{2}\right) v\right) d v d u=\left(1+q^{-3 s+1}\right) .
$$

Hence we have

Proposition 6. For the unramified additive character $\psi_{\sigma_{1}}$ associated to $\sigma_{1}=(0,1,1,0)$, we have

$$
I\left(f_{s}, \psi_{\sigma_{1}}\right)=\frac{\zeta_{v}^{2}(3 s-1)}{\zeta_{v}(3 s) \zeta_{v}(6 s-2) \zeta_{v}(9 s-3)}
$$

The following Corollary gives the evaluation of the exponential sum in Lemma 2 when $a \in \mathcal{O}^{\times, 3}$.

Corollary 3. With the notations as in above, we have

$$
S(a)=\sum_{\xi, \zeta, \nu \in \mathbb{F}_{q}^{\times} ; \mu \in \mathbb{F}_{q}} \psi_{\circ}\left(\left[a \xi^{3}-\mu^{3}-\zeta^{2}+\zeta-3 \mu \zeta\right] \pi^{-1} \nu\right)=3 q
$$

when $a \in \mathcal{O}^{\times, 3}$.

Proof. When $a=b^{3} \in \mathcal{O}^{\times, 3}$, it is easy to check that the vectors $\sigma_{3}(a)=$ $(1,0,0, a)$ and $\sigma_{1}=(0,1,1,0)$ are in the same $G L(2, \mathcal{O})$-orbit on the prehomogeneous space $(G L(2), V)$. More precisely, we may choose

$$
g=\left(\begin{array}{cc}
-b & 1 \\
-(1+\omega) b & (1+\omega) \omega^{2}
\end{array}\right) \in G L(2, \mathcal{O})
$$

so that $g \cdot(1,0,0, a)=(0,1,1,0)$. Hence for unramified data, we have

$$
I\left(f_{s}^{\circ}, \psi_{\sigma_{3}(a)}\right)=I\left(f_{s}^{\circ}, \psi_{\sigma_{1}}\right) .
$$

By Propositions 4 and part (1) of Proposition 3, we obtain

$$
\frac{\left(1-q^{-3 s}\right)\left(q^{-9 s+1}-q^{-18 s+4}\right)(S(a)-3 q)}{\left(1-q^{-9 s+3}\right)}=0 .
$$

Hence we have $S(a)=3 q$. 
5.3. Case: $\sigma_{2}=(0,1,0, a)$. Here we assume that $a$ is a unit in $F$ such that $x^{2}+a$ is irreducible over $F$. The integral we shall compute is

$$
I=\int_{F^{5}} f_{s}\left(n^{-}(x, y,-z,-u,-v)\right) \psi_{\circ}(a v+y) d n .
$$

A similar type of calculation works in this case, which gives us

$$
\begin{aligned}
I= & \int_{F^{3}} f_{s}\left(n^{-}(x, y,-z)\right) \psi_{\sigma_{2}}(y) d z d x d y \\
& \cdot\left(1+\int_{|u| \leq 1 ;|v|>1}|v|^{-3 s+1} \psi_{\sigma_{2}}\left(\left(a+u^{2}\right) v\right) d v d u\right) .
\end{aligned}
$$

Further, we have

$$
1+\int_{|u| \leq 1 ;|v|>1}|v|^{-3 s+1} \psi_{\sigma_{2}}\left(\left(a+u^{2}\right) v\right) d v d u=\frac{\left(1-q^{-6 s+2}\right)}{\left(1-\chi_{a}(q) q^{-3 s+1}\right)}
$$

where $\chi_{a}(q)=\left(\frac{-a}{q}\right)$, the quadratic character. Therefore we obtain

Proposition 7. Let a be a unit in F. For the additive character $\psi_{\sigma_{2}}$ with $\sigma_{2}=(0,1,0, a)$, we have

$$
I\left(f_{s}, \psi_{\sigma_{2}}\right)=\frac{\zeta_{v}(3 s-1) L_{v}\left(3 s-1, \chi_{a}\right)}{\zeta_{v}(3 s) \zeta_{v}(6 s-2) \zeta_{v}(9 s-3)}
$$

where $\chi_{a}$ is the character determined by $F\left(a^{\frac{1}{2}}\right) / F$ via the local class field theory.

\section{The Proof of Theorem 2.}

Let $F$ be a number field. Let $\mathfrak{p}$ be a prime ideal in $\mathcal{O}_{F}$ and $v$ be the corresponding place. Then $F_{v}=F_{\mathfrak{p}}$ is the local field associated to $\mathfrak{p}$ and $v$.

Part (1) and (2) of Theorem 2 follow directly from Proposition 4 and 5, respectively. In the following, we are going to prove part (3) and (4) of Theorem 2.

By the assumption of part (4) of Theorem 2, the base field $F$ contains the third root of unity, denoted by $\omega$. Given a $G L(2, \mathbb{A})$-orbit $\sigma_{4}=(1,0, b, a)$ of type 4 , the field $K$ generated by one of the roots of the irreducible polynomial $x^{3}+b x-a$ is a cubic extension over the base field $F$. If $\mathfrak{p}$ is unramified over $K$, then one has from $[\mathrm{CaFr}]$ that

$$
\mathfrak{p} \mathcal{O}_{K}= \begin{cases}\mathfrak{P}_{1} \mathfrak{P}_{2} \mathfrak{P}_{3}, & f_{1}=f_{2}=f_{3}=1, \\ \mathfrak{P}_{1} \mathfrak{P}_{2}, & f_{1}=1, f_{2}=2, \\ \mathfrak{P}, & f=3 .\end{cases}
$$


Correspondingly, one has

$$
K \otimes_{F} F_{\mathfrak{p}}=\left\{\begin{array}{l}
F_{\mathfrak{p}} \oplus F_{\mathfrak{p}} \oplus F_{\mathfrak{p}}, \\
F_{\mathfrak{p}} \oplus F_{\mathfrak{p}}(\alpha), \\
K_{\mathfrak{P}} .
\end{array}\right.
$$

Note that $F_{\mathfrak{p}}(\alpha)$ is an unramified quadratic extension over $F_{\mathfrak{p}}$ with $\alpha^{2}=$ $-c \in \mathcal{O}_{v}^{\times}$, and $K_{\mathfrak{P}}$ is an unramified cubic extension over $F_{\mathfrak{p}}$. Since $\omega \in F_{\mathfrak{p}}$, $K_{\mathfrak{P}}$ is a cyclic extension. Hence $K_{\mathfrak{P}}=F_{\mathfrak{p}}(\tau)$, where $\tau$ is one of the roots of $x^{3}-d$ with $d$ a unit in $F_{v}$.

Following the formulas in $\S 2$ of [Wri] and in Chapter 4 of [Jac], we can easily find elements $g_{v, 1}, g_{v, 2}, g_{v, 3} \in G L\left(2, \mathcal{O}_{v}\right)$ such that $g_{v, 1} \circ(1,0, b, a)=$ $(0,1,1,0)$ if $K \otimes_{F} F_{v}=F_{v} \oplus F_{v} \oplus F_{v}, g_{v, 2} \circ(1,0, b, a)=(0,1,0, c)$ if $K \otimes_{F} F_{v}=$ $F_{v} \oplus F_{v}(\alpha)$, and $g_{v, 3} \circ(1,0, b, a)=(1,0,0, d)$ if $K \otimes_{F} F_{v}=F_{v}(\tau)$, respectively, for all the finite places $v$ of $F$ outside of a finite subset $S$ of places of $F$ determined by the orbits $(1,0, b, a),(1,0,0, d)$, and $(0,1,0, c)$.

Because for the unramified section $f_{s, v}$, the Fourier coefficients with respect to $\psi_{\circ}(\langle\sigma, \cdot\rangle)$ and $\psi_{\circ}\left(\left\langle g_{v} \circ \sigma, \cdot\right\rangle\right)$ for $g_{v} \in G L(2, \mathcal{O})$ are equal to each other, the fourth part of Theorem 2 follows from Corollary 2 and Proposition 6 and 7 in $\S 5$.

Finally we shall prove the third part of Theorem 2. In this case we do not assume that $F$ contains the third root of unity. For each unramified place $v$ of $F$, when $F_{v}$ does not contain the third root of unity, $K \otimes_{F} F_{v}=F_{v} \times F_{v}\left(d^{\frac{1}{2}}\right)$, otherwise either $K \otimes_{F} F_{v}=F_{v} \oplus F_{v} \oplus F_{v}$ if $a$ is a cube at $v$ or $K \otimes_{F} F_{v}=F_{v}\left(a^{\frac{1}{3}}\right)$ if $a$ is not a cube. Similarly, we can reduce the computation of the local integral in this case to the three cases of the computations we made in $\S 5$. Hence the proof of Theorem 2 is completed.

\section{Proof of Lemma 2.}

This last section is devoted to the proof of Lemma 2 when $a \notin \mathcal{O}^{\times, 3}$. This means we are going to show that the multivariable cubic exponential sums

$$
S(a)=\sum_{\xi, \zeta, \nu \in \mathbb{F}_{q}^{\times} ; \mu \in \mathbb{F}_{q}} \psi_{\circ}\left(\left[a \xi^{3}-\mu^{3}-\zeta^{2}+\zeta-3 \mu \zeta\right] \pi^{-1} \nu\right)=0
$$

for $a \notin \mathcal{O}^{\times, 3}$. Here we assume that $a \in \mathbb{F}_{q}^{\times}$and $q \equiv 1(\bmod 3)$. When $a \in \mathcal{O}^{\times, 3}, S(a)=3 q$ as stated in Corollary 3 in $\S 5.2$.

The idea is to evaluate explicitly the following sum

$$
\sum_{a \in \mathbb{F}_{q}^{\times}} S(a)=\sum_{a, \xi, \zeta, \nu \in \mathbb{F}_{q}^{\times} ; \mu \in \mathbb{F}_{q}} \psi_{\circ}\left(\left[a \xi^{3}-\mu^{3}-\zeta^{2}+\zeta-3 \mu \zeta\right] \pi^{-1} \nu\right)
$$


in two different ways.

In one way, it is easy to see by the property that $S\left(a b^{3}\right)=S(a)$ for $b \in \mathbb{F}_{q}^{\times}$ that

$$
\sum_{a \in \mathbb{F}_{q}^{\times}} S(a)=\frac{q-1}{3}\left[S(1)+S(\alpha)+S\left(\alpha^{2}\right)\right]
$$

where $\left\{1, \alpha, \alpha^{2}\right\}$ is a set of representatives for the cosets of $\mathbb{F}_{q}^{\times} / \mathbb{F}_{q}^{\times, 3}$.

In the other way, we have:

Lemma 4. Let a be a unit of $F$. Then the sum

$$
\sum_{a \in \mathbb{F}_{q}^{\times}} S(a)=q(q-1)
$$

Proof. First we notice that

$$
\begin{aligned}
\sum_{a \in \mathbb{F}_{q}^{\times}} S(a)= & \sum_{\zeta, \nu \in \mathbb{F}_{q}^{\times} ; \mu \in \mathbb{F}_{q}} \psi_{\circ}\left(\left[\mu^{3}-\zeta^{2}+\zeta+3 \mu \zeta\right] \pi^{-1} \nu\right) \sum_{a, \xi \in \mathbb{F}_{q}^{\times}} \psi_{\circ}\left(a \xi^{3} \pi^{-1} \nu\right) \\
= & -(q-1) \sum_{\zeta, \nu \in \mathbb{F}_{q}^{\times} ; \mu \in \mathbb{F}_{q}} \psi_{\circ}\left(\left[\mu^{3}-\zeta^{2}+\zeta+3 \mu \zeta\right] \pi^{-1} \nu\right) \\
=- & (q-1) \sum_{\nu \in \mathbb{F}_{q}^{\times} ; \zeta, \mu \in \mathbb{F}_{q}} \psi_{\circ}\left(\left[\mu^{3}-\zeta^{2}+\zeta+3 \mu \zeta\right] \pi^{-1} \nu\right) \\
& +(q-1) \sum_{\nu \in \mathbb{F}_{q}^{\times} ; \mu \in \mathbb{F}_{q}} \psi_{\circ}\left(\mu^{3} \pi^{-1} \nu\right) .
\end{aligned}
$$

Since the last sum is zero, we obtain that

$$
\begin{gathered}
\sum_{a \in \mathbb{F}_{q}^{\times}} S(a)=-(q-1) \sum_{\nu \in \mathbb{F}_{q}^{\times} ; \zeta, \mu \in \mathbb{F}_{q}} \psi_{\circ}\left(\left[\mu^{3}-\zeta^{2}+\zeta+3 \mu \zeta\right] \pi^{-1} \nu\right) \\
=-(q-1)\left[(q-1) N\left(\zeta^{2}-(1+3 \mu) \zeta-\mu^{3}\right)\right. \\
\left.\quad-N^{-}\left(\zeta^{2}-(1+3 \mu) \zeta-\mu^{3}\right)\right]
\end{gathered}
$$

where $N\left(\zeta^{2}-(1+3 \mu) \zeta-\mu^{3}\right)$ denotes the number of the solutions of the equation $\zeta^{2}-(1+3 \mu) \zeta-\mu^{3}=0$ over $\mathbb{F}_{q}^{2}$ and $N^{-}\left(\zeta^{2}-(1+3 \mu) \zeta-\mu^{3}\right)$ denotes the number of the points in $\mathbb{F}_{q}^{2}$ which are not a solution of the equation $\zeta^{2}-(1+3 \mu) \zeta-\mu^{3}=0$. Thus we have

$$
\sum_{a \in \mathbb{F}_{q}^{\times}} S(a)=q^{2}(q-1)-q(q-1) N\left(\zeta^{2}-(1+3 \mu) \zeta-\mu^{3}\right) .
$$


Let $\phi_{q}(a)$ be the quadratic residue character associated to $q$. Then the number $N\left(\zeta^{2}-(1+3 \mu) \zeta-\mu^{3}\right)$ can be calculated as follows.

$$
\begin{aligned}
N\left(\zeta^{2}-(1+3 \mu) \zeta-\mu^{3}\right) & =q+\sum_{\mu \in \mathbb{F}_{q}} \phi_{q}\left(1+6 \mu+9 \mu^{2}+4 \mu^{3}\right) \\
& =q+\sum_{\mu \in \mathbb{F}_{q}} \phi_{q}\left((\mu+1)^{2}(4 \mu+1)\right) \\
& =q+\sum_{\mu \in \mathbb{F}_{q}} \phi_{q}\left(\mu^{2}(4 \mu-3)\right) \\
& =q+\sum_{\mu \in \mathbb{F}_{q}^{\times}} \phi_{q}(4 \mu-3) \\
& =q-\phi_{q}(-3)+\sum_{\mu \in \mathbb{F}_{q}} \phi_{q}(4 \mu-3) \\
& =q-1
\end{aligned}
$$

since $\phi_{q}(-3)=1$ (as we assume that $\left.q \equiv 1(\bmod 3)\right)$ and the last character sum is zero. Therefore we obtain

$$
\sum_{a \in \mathbb{F}_{q}^{\times}} S(a)=q^{2}(q-1)-q(q-1)^{2}=q(q-1),
$$

as expected.

According to the two different ways to evaluate the sum

$$
\sum_{a \in \mathbb{F}_{q}^{\times}} S(a)
$$

and $S(1)=3 q$, we have

$$
S(\alpha)+S\left(\alpha^{2}\right)=3 q-S(1)=0 .
$$

It remains to show that $S(\alpha)=S\left(\alpha^{2}\right)$. By Lemma 1 in [Wri1], these two vectors $\sigma_{3}^{\prime}=(1,0,0, \alpha)$ and $\sigma_{3}^{\prime \prime}=\left(1,0,0, \alpha^{2}\right)$ are in the same $G L(2)$-orbit on the prehomogeneous space $V$ (over either the local field or the residue field). In fact, it is easy to check that both $\sigma_{3}^{\prime}$ and $\sigma_{3}^{\prime \prime}$ are in the same $G L(2, \mathcal{O})$-orbit (transformed by $\left.\left(\begin{array}{ll}0 & 1 \\ \alpha & 0\end{array}\right)\right)$. Hence, for unramified data, we have

$$
I\left(f_{s}^{\circ}, \sigma_{3}^{\prime}\right)=I\left(f_{s}^{\circ}, \sigma_{3}^{\prime \prime}\right) .
$$

Following Corollary 2 in $\S 5.1$, we must have

$$
S(\alpha)=S\left(\alpha^{2}\right) .
$$


This and (33) imply that $S(a)=0$ when $a \notin \mathcal{O}^{\times, 3}$ and complete the proof of Lemma 2.

\section{References}

[Bra] R. Brauer, On the zeta-function of algebraic number fields, Amer. J. Math., 69 (1947), 243-250; 72 (1950), 739-746.

[Car] R. Carter, Simple groups of Lie type, John Wiley and Sons, 1972.

[CaFr] J. Cassels and A. Frohlich, Algebraic number theory, Academic Press, 1967.

[DaWr] B. Datskovski and D. Wright, The adelic zeta function associated to the space of binary cubic forms, II: Local theory, J. Reine Angw. Math., 367 (1986), 27-75.

[Fli] Y.Z. Flicker, The adjoint representation L-function for $G L(n)$, Pacific J. Math., 154(2) (1992), 231-244.

[Fro] A. Frohlich, Algebraic number fields, Academic Press, 1977.

[GeJa] S. Gelbart and H. Jacquet, A relation between automorphic representations of $G L(2)$ and $G L(3)$, Ann. Sci. Ec. Norm. Sup., 11 (1978), 471-542.

[Gin] D. Ginzburg, A Rankin-Selberg integral for the adjoint representation of GL(3), Invent. Math., 105 (1991), 571-188.

[GRS] D. Ginzburg, S. Rallis and D. Soudry, Cubic correspondences arising from $G_{2}$, preprint, 1995.

[Hec] E. Hecke, Lectures on the theory of algebraic numbers, Springer-Verlag, 1981.

[Igu] J. Igusa, Arithmetic of a singular invariant, Amer. J. Math., 110 (1988), 197-233.

[Jac] N. Jacobson, Basic algebra I, (second edition), W.H. Freeman and Company, New York, 1985.

[JaSh] H. Jacquet and J. Shalika, On Euler products and the classification of automorphic representations I, Amer. J. Math., 103 (1981), 499-558.

[JaZa] H. Jacquet and D. Zagier, Eisenstein series and the Selberg trace formula II, Trans. of Amer. Math. Soc., 300(1) (1987), 1-48.

[Jia] D. Jiang, Poles of certain Eisenstein series of $G_{2}$, unpublished notes.

[MoWa] C. Moeglin and J.-L. Waldspurger, Modeles de Whittaker degeneres pour des groupes p-adiques, Math. Z., 196 (1987), 427-452.

[Sav] G. Savin, An analogue of the Weil representation for $G_{2}$, J. Reine Angw. Math., 434 (1993), 115-126.

[Shi] G. Shimura, On the holomorphy of certain Dirichlet series, Proc. London Math. Soc., 31 (1975), 79-98.

[Sie] C.L. Siegel, Uber die analytische theorie der quadatischen formen, Ann. of Math., 36 (1935), 527-606.

[Ste] R. Steinberg, Lectures on Chevalley groups, Lecture Notes, Yale University, 1968.

[Wri] D. Wright, The adelic zeta function associated to the space of binary cubic forms, I: Global theory, Math. Ann., 270 (1985), 503-534.

[Wri1] Cubic character sums of cubic polynomials, Proc. of Amer. Math. Soc., 100(3) (1987), 409-413. 
[WrYu] D. Wright and A. Yukie, Prehomogeneous vector spaces and field extensions, Invent. Math., 110 (1992), 283-314.

Received May 22, 1996 and revised December 18, 1996.

YALE UNIVERSITY

New Haven, CT 06520-8283

AND

The Ohio State University

Columbus, OH 43210 